\title{
A novel framework for modelling the 3D mesostructure of steel fibre reinforced concrete
}

\section{Sadjad Naderi, Mingzhong Zhang*}

Department of Civil, Environmental and Geomatic Engineering, University College London, London WCIE 6BT, UK

Abstract: This paper presents a novel method for modelling the three-dimensional four-phase mesostructure of steel fibre reinforced concrete (SFRC) consisting of coarse aggregates, mortar, interfacial transition zone and fibres, which provides several advanced features. It enables constructing various types of short discrete fibre like straight, spiral and hooked-end fibres with one- or three-dimensional elements while configuring their sizes and orientations. A key aspect of the approach is the capability of generating realistic-shaped aggregates with size- and shape-adjustability among fibres in a stochastic domain based on the relation of Voronoi and Delaunay structures. The mesostructure model is a powerful simulation tool for characterisation of such heterogenous materials because of the strong structural controllability, the algorithm's flexibility, the low computational cost and the productivity for many random samples in a statistical framework. To show the feasibility, the models were successfully implemented in a finite element case study to evaluate the elastic modulus of SFRC.

Keywords: Irregularly shaped aggregate; Interfacial transition zone; Fibre reinforcement; RVE; Voronoi tessellation; Finite element analysis

\section{Introduction}

Concrete as a common construction material has a great variety of applications due to its good strength and durability. Material properties like mechanical strength and transport properties of concrete have been always interesting technical and scientific subject of study. However, a reliable prediction of such properties is still a significant challenge because concrete is an extremely complex heterogeneous material. The heterogeneity mainly depends on four phases detected in mesostructure of concrete, the size of which might vary from submicro/micro- to macro-scale [1]. They are dominant structural features in local damage evolution and mass transport $[2,3]$ and each phase plays a key role in the macroscopic behaviour of concrete [4]. The first phase is irregular shaped aggregates which are randomly distributed within the second phase of mortar. The previous studies have proved that the shape and size of the coarse aggregates have significant influence on workability, mechanical performance and durability of concrete [5]. The interfacial layer between aggregate and mortar as the third phase is called interfacial transition zone (ITZ), which would substantially affect the properties of concrete $[6,7]$. Fibres as the fourth phase can be added to unreinforced concrete in order to improve its excessive brittleness and weak behaviour in tension. Fibrous materials can also enhance shear strength and energy absorption capacity [8,9]. All these characteristics make fibre a very important structural element beside the other three phases. So, it is critical to consider the interaction of all of them in the analysis of properties of fibre reinforced concrete (FRC). In terms of numerical method, a mesoscale model framework can provide a more realistic representation of the material compared to the homogenous model [10]. In an ideal mesostructure model, all geometric features such as aggregates and fibres with the actual structural characteristics should be explicitly simulated. However, covering all effective properties in an irregular system

\footnotetext{
* Corresponding author.

E-mail addresses: s.naderi@ucl.ac.uk (S. Naderi), mingzhong.zhang@ucl.ac.uk (M. Zhang)
} 
causes some modelling difficulties. Typical problems can be resolved through keeping computational volume low and replicating geometry with more natural features.

Regarding unreinforced concrete, one of the main simulation challenges is how to deal simultaneously with shape, size and distribution heterogeneity of aggregates. Furthermore, the ITZ structure is influenced by aggregate geometry in the sense of modelling complexity as it is a layer around aggregate. Generally, two computational approaches have been used to simulate the aggregate particles. The first is the image-based model that replicates a mesostructure by scanning a real sample, e.g. using X-ray computed tomography (CT). The approaches enable us to model a mesostructure with many realistic details [11-13]. However, they require expensive computations to produce a limited number of models which only correspond to features of a specific image $[14,15]$. In contrast, the simplified geometries have been extensively employed as the second method to overcome the complexities [16-18]. For example, a regular geometry model (e.g. sphere or ellipsoid) was used for the aggregate regardless of its natural irregular shape [19, 20]. Most of these works suffered from resolving non-overlapping during the reconstructing process. The implementation of simplifications might decrease the complexities, but at the same time, it sacrifices accuracy.

Regarding reinforced concrete, the modelling process becomes more difficult if fibrous materials like short discrete fibres are added to concrete with random orientation and distribution [21]. Heterogeneity and computational cost are intensified due to the composition of multiple stochastic systems created by fibres, aggregates and ITZs. Fibres should be placed among the irregular aggregates randomly distributed while examining intersections and size distribution of different components. As mentioned earlier, the simplification has been mostly used as a solution to the high complexity in the previous studies. In some cases, the model of FRC was simplified to two dimensions [22-23] and the mortar matrix and coarse aggregate were treated as the homogenous continuum [24-26]. Similarly, the elastic properties of the spherical aggregate and the ITZ surrounding each aggregate were homogenised [9, 27]. Or again, the X-ray CT was employed to reconstruct the mesostructure of FRC [23]. Due to the complications, there have been very limited studies to explicitly model fibres and ITZ in addition to other phases in FRC and a comprehensive method for simulating such multi-scale random behaviour of FRC is still lacking. It is even more essential for steel fibres as the most widely used in civil infrastructures amongst all types of fibre such as natural, carbon and glass fibres [28]. To the best of the authors' knowledge, there has not been such integrated mesostructure model which possesses all the following features together: (1) full control shape, orientation and size of fibres; (2) generation of irregular aggregate based on arbitrary shape, size distribution; and (3) easy approach to model ITZ and mortar matrix. To address these characteristics, a new algorithm is required to model steel fibre reinforced concrete (SFRC) with high efficiency in terms of computational cost and accuracy. In this study, the primary objective is to develop such an advanced algorithm with the features mentioned.

Regarding the virtual generation of fibrous media, some available approaches have been proposed to simulate the fibres based on the existing mesh. For instance, the fibres were modelled in the finite element (FE) analysis as two-nodded one-dimensional (1D) elements bonded to the matrix mesh [29]. In such models, the fibre geometry is normally constructed through a string of nodes, elements or predefined cells. It makes the configurations of orientation, number and size be restricted to the matrix mesh. Another difficulty may 
raise when the fibres are initially generated using a set of points and the model is then meshed [25]. In this case, the mesh quality might be affected by a large number of randomly distributed short fibres. Moreover, additional phase like aggregates cannot be easily inserted in a matrix with the presence of the fibres prescribed. As a solution the Delaunay triangulation technique was implemented to improve the meshing process, which allowed the 1D fibres to be appropriately linked to the three-dimensional (3D) matrix elements [30]. In the current work, the Delaunay triangulation strategy will be employed to generate short fibres at the desired locations according to the model framework proposed.

In the authors' prior publication [31], an integrated modelling framework was presented for generating the 3D virtual mesostructure of the particulate media using Voronoi and splining methods. The proposed approach could control the shape and size of the irregulate particles (coarse aggregates) in the random distribution system. The shape parameters (e.g. sphericity, roundness and convexity) and the sieve analysis as the inputs were used to configure the $3 \mathrm{D}$ concrete model based on the experimental data. The advantages of the simulation techniques implemented were fully discussed. In continuation of this work, the present paper aims to further develop the previous algorithm to add ITZ and fibres in the 3D particulate mesostructure model for FRC. The novel four-phase 3D mesoscale model of SFRC offers new opportunities, which enables us to investigate material behaviour influenced by steel fibres beside particles and ITZ embedded in mortar. These structural parameters can be quantitatively characterised concerning their shapes, sizes and distribution modes as well as fibre orientation. Three typical steel fibres including straight, hooked-end and spiral fibres are considered as examples for simulations, showing the ability of the framework to deal with different profiles commonly used in FRC. The geometries can be made by 1D and 3D elements to be compatible with different material models and simulation techniques. The dimensions of all the fibres are completely adjustable. The ITZ model is characterised by its thickness. The boundary of solid model geometry is first generated as triangulated surfaces and then the solid mesh is performed among the surfaces. Fig. 1 shows an overview of the modelling process and the main features of each step as a flow chart. First, the straight, spiral and hookedend fibres are modelled. Then, the coarse aggregates are generated using a technique based on the relationship between Delaunay triangulation and Voronoi tessellation. The shape and size of aggregates are adjusted by the approach presented in [32]. Afterwards, the ITZ are constructed relying on aggregate geometry. In the final step, mortar and SFRC model are produced by solid mesh through the triangulated surfaces of other components. It is worth mentioning that all the algorithms are coded in MATLAB. To trial the model feasibility and efficiency, the equivalent elastic moduli of some SFRC models are evaluated using FE method.

\section{Fibre generation}

Fibres are built using a set of coupled points which are systematically distributed. The system to distribute the points has a key role in the modelling process. It is designed based on the Delaunay triangulation strategy and the Voronoi tessellation method as the basis of generation of particles in the next steps. The systematic point distribution provides conditions for constructing different phases with no intersection. A geometric intersection problem is a typical issue in the simulation of a complex solid structure. Most of the algorithms existed suffer from either avoiding or fixing existed overlaps. The approach proposed significantly reduces simulation complications. In the following sections, the methodology will be described for simulating the 
straight fibres with the circular cross-section made by 1D elements. Then, the models of hooked-end and spiral fibres will be presented with reference to the model approach of straight fibre. Finally, further development to create fibre with triangulated surfaces will be discussed which is useful in modelling fibre as a 3D solid object.

\subsection{Straight fibre}

\subsubsection{Geometric essentials}

Firstly, it is essential to introduce the important geometric parameters and principles in the proposed model. Also, the constraints on the positions of fibres should be clearly stated. Some definitions like Delaunay triangulation and Voronoi diagram are well-known and have been frequently repeated in the literature. However, they are presented below for the paper completeness:

- Circumcircle: the unique circle which passes all three vertices of a triangle (see Fig. 2). The centre of the circumcircle is called the circumcentre, and the radius of the circle is named the circumradius.

- Circumsphere: with a similar definition as circumcircle but it is specified by a sphere and a tetrahedron instead of circle and triangle, respectively.

- Delaunay criterion: for a two-dimensional (2D) set of points, a Delaunay triangulation of these points ensures the circumcircle associated with each triangle does not contain any other point in its inside. Fig. 2a shows an example of Delaunay triangulations composed of four points, two triangles and the related circumcircles. The triangles share two vertices of $V_{1}$ and $V_{2}$. The Delaunay triangulation satisfies the empty circumcircle criterion. On the contrary, Fig. $2 \mathbf{b}$ illustrates an example which is not considered as a Delaunay triangulation. By fulfilling the empty circumcircle property, well-shaped triangles with the large internal angles are preferred to ones with smaller angles. The triangles in the non-Delaunay triangulation have sharper at vertices $V_{1}$ and $V_{2}$. Moreover, among the points distributed, three closest points are the best candidates for making a triangle [32]. These two features, i.e. well-shaped triangles and the nearestneighbour relation, are important implications that can be effectively used in the fibre generation. The description mentioned above can be similarly extended to a 3D set of points composed of tetrahedra.

- Relationship between Delaunay triangulation and Voronoi diagram: the fundamental properties of the Voronoi tessellation method have been reported many times in the literature. The reader is referred to [33] for a comprehensive review. Here, more focus is placed on the properties required. A Voronoi diagram can correspond to the Delaunay triangulation. The circumcentres of Delaunay triangles/tetrahedra are the vertices of the Voronoi cells as exemplified by Fig. 3a and b. In a 2D/3D case, the Voronoi vertices are connected via edges/faces, if two triangles/tetrahedrons share an edge/face in the Delaunay triangulation. If so, their circumcentres can be connected with an edge/face in the Voronoi tessellation [34]. In other words, the centres of two adjacent Voronoi cells are connected by an edge/face of a triangle/tetrahedron for classic Voronoi tessellation (Fig. 3c). The shared edge/plane is the perpendicular bisector of a line segment which connects the centres of two adjacent cells.

- Shortest distance between two line segments: to find the distance, the geometric method presented in [35] is used. At first, the closest points on the lines should be detected. As shown in Fig. 4, these unique points can be located on the line either between endpoints or anywhere else outside the range of segments on the extended infinite lines (lines 1 and 2). The determination of the shortest distance can be implied by the 
following method and with the assistance of Fig. 4. In 3D, the line segments $S_{1}$ and $S_{2}$ with endpoints $\left\{P_{0}, P_{1}\right\}$ and $\left\{Q_{0}, Q_{1}\right\}$ are represented by:

$$
\begin{array}{ll}
P(s)=P_{0}+s\left(P_{1}-P_{0}\right)=P_{0}+s \boldsymbol{u} & 0 \leq s \leq 1 \\
Q(t)=Q_{0}+t\left(Q_{1}-Q_{0}\right)=Q_{0}+t \boldsymbol{v} & 0 \leq t \leq 1
\end{array}
$$

The vector $\boldsymbol{w}$ with start points on line 1 and an endpoint on line 2 can be defined as:

$$
\begin{aligned}
& \boldsymbol{w}(s, t)=P(s)-Q(t)=\left(P_{0}-Q_{0}\right)+s \boldsymbol{u}-\boldsymbol{t} \boldsymbol{v} \\
& \text { if } \boldsymbol{w}_{\mathbf{0}}=P_{0}-Q_{0} \Rightarrow \boldsymbol{w}(s, t)=\boldsymbol{w}_{\mathbf{0}}+s \boldsymbol{u}-\boldsymbol{t} \boldsymbol{v}
\end{aligned}
$$

At the closest points $P\left(s_{c}\right)$ and $Q\left(t_{c}\right)$ on the infinite lines, the vector $\boldsymbol{w}_{\boldsymbol{c}}$ is uniquely perpendicular to the vectors $\boldsymbol{u}$ and $\boldsymbol{v}$. So, it satisfies two linear equations at the same time:

$\boldsymbol{u} \cdot \boldsymbol{w}_{\boldsymbol{c}}=0, \quad \boldsymbol{v} \cdot \boldsymbol{w}_{\boldsymbol{c}}=0$

By substituting $\boldsymbol{w}_{\boldsymbol{c}}=\boldsymbol{w}_{\mathbf{0}}+s_{c} \boldsymbol{u}-t_{c} \boldsymbol{v}$ into Eq. (3) it gives:

$\left\{\begin{array}{l}(\boldsymbol{u} \cdot \boldsymbol{u}) s_{c}-(\boldsymbol{u} \cdot \boldsymbol{v}) t_{c}=-\boldsymbol{u} \cdot \boldsymbol{w}_{\mathbf{0}} \\ (\boldsymbol{v} \cdot \boldsymbol{u}) s_{c}-(\boldsymbol{v} \cdot \boldsymbol{v}) t_{c}=-\boldsymbol{v} \cdot \boldsymbol{w}_{\mathbf{0}}\end{array}\right.$

If $a=\boldsymbol{u} \cdot \boldsymbol{u}, b=\boldsymbol{u} \cdot \boldsymbol{v}, c=\boldsymbol{v} \cdot \boldsymbol{v}, d=\boldsymbol{u} \cdot \boldsymbol{w}$ and $e=\boldsymbol{v} \cdot \boldsymbol{w}$, then $s_{c}$ and $t_{c}$ are obtained and expressed as:

$$
\begin{aligned}
s_{c} & =\frac{b e-c d}{a c-b^{2}} \\
t_{c} & =\frac{a e-b d}{a c-b^{2}}
\end{aligned}
$$

If the dominator $a c-b^{2}=0$, two lines are parallel and the distance between the lines is constant. In this case, the closest points can be selected based on any point on the line segments like the endpoints. For instance, at $P_{0}, s_{c}$ is equal to zero and according to Eq. (4), $t_{c}=d / b=e / c$.

If the obtained $s_{c}$ and $t_{c}$ are both within the range of zero to one, they are accepted as the closest point for the line segments and the shortest distance is $\left|\boldsymbol{w}\left(s_{c}, t_{c}\right)\right|$. But if they are not, the new points are determined based on minimizing the length of $\boldsymbol{w}(s, t)$ or in other words, $|\boldsymbol{w}|^{2}$. Basically, $|\boldsymbol{w}|^{2}$, which can be expanded as $\boldsymbol{w} \cdot \boldsymbol{w}=\left(\boldsymbol{w}_{\mathbf{0}}+s \boldsymbol{u}-t \boldsymbol{v}\right) \cdot\left(\boldsymbol{w}_{\mathbf{0}}+s \boldsymbol{u}-t \boldsymbol{v}\right)$, is a quadratic function of $s$ and $t$. It defines a paraboloid on the plane $(s, t)$ with a minimum at $C=\left(s_{c}, t_{c}\right)$, as shown in Fig. $4 \mathbf{b}$. The minimum $C$ lies outside of the subregion $\mathbb{G}$, when $s_{c}$ and $t_{c}$ are out of the range $(0,1)$. In these cases, it can be concluded that for the line segments, if $s_{c}$ or $t_{c}$ is less than 0 , then it is set 0 ; and, if $s_{c}$ or $t_{c}$ is greater than 1 , it is set 1 .

- Parent points: the unit fibre model is generated based on a straight line between a start and an endpoint (Fig. 5a).

- Length of fibre: the distance between parent points are adjusted according to fibre length $L_{f}$ as the numerical input (Fig. 5a).

- Child points: the points are produced on each line which divide the line into equal sub-lines (Fig. 5a).

- Fibre generator points (FGP): a set of points include parent and child points.

- 1D-element: in the presence of child points, a sub-line represents a 1D element (Fig. 5a). Otherwise, the line between the parent points is interpreted as the element. The nodes of elements are the points associated with the corresponding line/subline. $L_{e}$ denotes the length of element for the straight fibre. 
- Distance between two fibres: the distance $D_{f}$ is the minimum distance allowed between two fibres (Fig. 5a). To avoid intersecting, $D_{f}$ must be greater than the diameter of the unit fibre.

- Number of fibres: this input parameter $N_{f}$ is obtained by the ratio of the total volume of fibres to the volume of one fibre.

- Domain dimensions: the model domain is cube-shaped with a side length of $D_{m}$ (Fig. 5b).

- Offset: $D_{o}$ is the minimum distance of all FGPs allowed from the free surfaces of the domain (Fig. 5b).

- Rule of connectivity: the FGPs will be used as the generator points of Voronoi tessellation. To manage the positions of the Voronoi cells, fibres and the corresponding 1D elements should pass through the centre of the cells. In other words, they should be lined up with the sides of the Delaunay triangles (refer to Fig. $\mathbf{3 b}$ and $\mathbf{c}$ ), which makes the positions of particles predictable to the positions of fibres in such a random structure, as the particle models are created depending on the geometry of the Voronoi cells. Fig. 6 is employed to clearly demonstrate the rule of connectivity among the FGPs according to the connectivity of the Delaunay triangles. Two possible states are shown in the figure for two sets of points which are connected based on the Delaunay criterion. In a Delaunay triangulation, three closest points are the best candidates for making a triangle or tetrahedron. Fig. 6a illustrates perfect links between the points because the distance between two adjacent points on fibre is not greater than $D_{f}$. Otherwise as shown in Fig. 6b, the points follow the criterion and get connected to a closer point which does not belong to the fibre structure. It causes discontinuity by making a wrong link through the fibre model in the structure of the Delaunay-based mesh. Thus, the rule of connectivity indicates that length of $L_{e}$ must be always less than $D_{f}$.

\subsubsection{Computer algorithm}

According to the descriptions above, the general layout of the algorithm is described. It follows the steps outlined below:

Step 1. The information related to the geometrical characteristics is set as the input parameters including $D_{m}$, $D_{o}, D_{f}, L_{f}$ and $N_{f}$.

Step 2. $L_{e}$ as input should be calculated in accordance with the rule of connectivity. If $L_{f} \geq D_{f}$, the minimum number of child points should be greater than $L_{f} / D_{f}$. If $L_{f}<D_{f}$, the number of child point can be set to zero.

Step 3. The parent points are randomly generated using the MATLAB function rand in the region determined by the offset from the cube's surfaces. The start point is produced and then endpoint with the distance $L_{f}$ is generated. In this study, the specific fibre orientation is not considered, and the fibres are modelled in random directions. However, it is worth mentioning that the fibre orientation can be simply applied to the code at this step by considering in the generation of the endpoint.

Step 4. The line made by parent points is checked to be consistent with the geometric condition of $D_{f}$. If the distance between fibres is less than $D_{f}$, the points are declined, and a new couple of points are generated as mentioned in Step 3.

Step 5. If required, the child points are produced. 
The outputs of the above algorithm are two matrices including nodal positions and element connectivity. Each node is identified by the related row number in the matrix. The element connectivity is defined by node IDs. Each element is recognised by the related row number in the array and the fibre's ID. The fibres are labelled in order of generation. In terms of programming, the IDs help to recall an element or node for different purposes.

As exemplified in Fig. 7, the fibres are embedded into the mesh structure generated based on the Delaunay criterion. The fibres are automatically aligned with the edges of the tetrahedrons through the mesh. The positions of fibre points dictated by this approach have provided the conditions to be consistent with the mesh. It should be noted that the Voronoi-based mesh presented here is only used to visualize the relationship between the generated fibre and Delaunay triangulation, and does not represent the final solid mesh.

\subsection{Spiral fibre}

\subsubsection{Geometric essentials}

- Circular spiral (helix) curve: it is a 3D curve as a well-known geometric feature that turns around an axis at a constant distance while moving parallel to the axis [36]. In mathematics, a helix can be defined using circular helix of radius $a$, and slope $b / a$ (or pitch $2 \pi b$ ), as illustrated in Fig. 8, with the following parametrization:

$$
\begin{aligned}
& x(t)=a \cos (t) \\
& y(t)=a \sin (t) \\
& z(t)=b t
\end{aligned}
$$

- Rotation matrix from axis and angle: it rotates points through angle $\theta$ counterclockwise about an axis in the direction of $\boldsymbol{u}$ and the origin of the Cartesian coordinate system. Given a unit vector $\boldsymbol{u}=\left(u_{x}, u_{y}, u_{z}\right)$, where $u_{x}{ }^{2}+u_{y}{ }^{2}+u_{z}{ }^{2}=1$, the rotation matrix $R$ is [37]:

$$
\left[\begin{array}{ccc}
\cos \theta+u_{x}^{2}(1-\cos \theta) & u_{x} u_{y}(1-\cos \theta)-u_{z} \sin \theta & u_{x} u_{z}(1-\cos \theta)+u_{y} \sin \theta \\
u_{y} u_{x}(1-\cos \theta)+u_{z} \sin \theta & \cos \theta+u_{y}{ }^{2}(1-\cos \theta) & u_{y} u_{z}(1-\cos \theta)-u_{x} \sin \theta \\
u_{z} u_{x}(1-\cos \theta)-u_{y} \sin \theta & u_{z} u_{y}(1-\cos \theta)+u_{x} \sin \theta & \cos \theta+u_{z}{ }^{2}(1-\cos \theta)
\end{array}\right]
$$

If it is multiplied by vector $v=(x, y, z)$ representing a point, then the matrix multiplication yields the result of rotating the point, as follows:

$$
\left[\begin{array}{c}
u_{x}\left(u_{x} x+u_{y} y+u_{z} z\right)(1-\cos \theta)+x \cos \theta+\left(-u_{z} y+u_{y} z\right) \sin \theta \\
u_{y}\left(u_{x} x+u_{y} y+u_{z} z\right)(1-\cos \theta)+y \cos \theta+\left(u_{z} x-u_{x} z\right) \sin \theta \\
u_{z}\left(u_{x} x+u_{y} y+u_{z} z\right)(1-\cos \theta)+z \cos \theta+\left(-u_{y} x+u_{x} y\right) \sin \theta
\end{array}\right]
$$

- Parent points: the start and endpoints as shown in Fig. 8a and $\mathbf{b}$ are interpreted as the parent points. The parent points produced by Eq. (6) are always on a line parallel to the axis.

- Child points: they are some points expressed by Eq. (6) on the spiral curve which connects 1D elements with the same length of $L_{e}$ (Fig. 8b). This length should be small enough to properly represent the curvature of the helix structure. Against the straight fibres, the rule of connectivity is not applied on $L_{e}$ because theses parent and child points will not be used for Voronoi tessellation.

- Length of fibre: the length of the axis is interpreted as the length of fibre $L_{f}$ (Fig. 8b). The distance between parent points also equals to $L_{f}$. 
- Number of fibres: to obtain $N_{f}$, the volume of unit fibre is calculated by the length of the spiral curve and the area of the cross-section. To ease computation, it can be estimated with the assistance of $1 \mathrm{D}$ elements.

- Distance between two fibres: $D_{f}$ between two spiral fibres can be measured with reference to their axes. Therefore, $D_{f}$ should be greater than the sum of $2 a$ and the diameter of fibre's cross-section.

- Offset: it is set up according to the position of the axis. If the offset value for the straight fibre model is $D_{o}$, it is $D_{o}+a$ for the spiral fibre.

- Vector of fibre: it is defined as a vector with the length $L_{f}$. It is located on and aligned with the axis. As shown in Fig. 8b, it connects two planes perpendicular to the fibre axis at the ends of the fibre.

\subsubsection{Computer algorithm}

The algorithm proposed to generate the randomly distributed spiral fibres in the representative volume elements (RVEs) of FRC contains three modules: (1) Axes Generator, (2) Single-Fibre Maker, and (3) Assembler. Fig. 9a schematically illustrates how these three modules interact to create the final model. Axes Generator constructs the fibre vectors representing the axis of fibres based on the algorithm of the straight fibres. However, the input parameters $D_{m}, D_{o}, D_{f}, L_{f}$ and $N_{f}$ should be determined according to the geometric features of spiral fibre. The outputs of this module are the matrices including the position of the endpoints on the axes (or line segments) and the vectors which connect the endpoints. The module of Single-Fibre Maker builds one fibre aligned along $Z-$ axis as shown in Fig. 9. For the fibre axis, one of the endpoints is at the origin $(0,0,0)$ and the other one is located on the positive part of $Z-$ axis. The parameters $a, b, L_{f}$ and $L_{e}$ are used as the inputs. The module's production is the matrix of the position of parent and child points in order of generation. The inputs to Assembler consist of the outputs two other modules. Assembler duplicates the fibre model generated by Single-Fibre maker, rotates it along a fibre vector created by Axes Generator and translate the rotated fibre to the position of the corresponding vector. This process is repeated for many times that equal to the number of vectors produced. Fig. 9 indicates that the rotation is performed based on the cross product of the fibre vector and the unit vector of $Z-$ axis $(\hat{\boldsymbol{z}}=(0,0,1))$. The cross product as the unit vector $\boldsymbol{u}$ and the angle $\theta$ are implemented into the rotation matrix according to Eq. (8). To translate, a vector between the origin and the start point of the related fibre vector is used. The final geometry model contains the data related to nodes and elements with a similar format to the straight fibre model. A sample of the final model generated using the algorithm is also presented in Fig. 9.

\subsection{Hooked-end fibre}

\subsubsection{Geometric essentials}

- Dimensions of fibre: the unit fibre is modelled by five-line segments. The dimensions of a fibre can be introduced to the code as inputs by four parameters including $L_{1}, L_{2}, L_{3}$ and $\alpha$ as indicated in Fig. 10a.

- Length of fibre: as shown in Fig. 10a, $L_{f}$ is equal to $2\left(L_{1}+L_{2} \cos (\alpha)\right)+L_{3}$.

- Number of fibres: $N_{f}$ is obtained based on the volume of the unit fibre which is calculated by multiplying the cross-section area and the total length specified as $2\left(L_{1}+L_{2}\right)+L_{3}$ (Fig. 10a).

- Coordinate system setup: as seen in Fig. 10a, for the unit fibre, one of the endpoints (point 1) is located at the origin of the Cartesian system and the other one (point 6) is relatively on the positive part of $Z$-axis. 
The points 3 is randomly generated around $Z$ - axis with the radius of $L_{2} \sin (\alpha)$. All points produced are in the same plane (Fig. 10b).

- Parent point: the couple points of $\{1,2\},\{2,3\},\{3,4\},\{4,5\}$ and $\{5,6\}$ are the parent points which indicate five segments (Fig. 10a).

- Child point: it is defined as previously stated but the number of points can change for each line segment. If required, they are produced between parent points. Therefore, the length of $L_{e}$ can vary for the lines with three different lengths. The rule of connectivity is also not applicable to $L_{e}$.

- Vector of fibre: it connects two endpoints (point 1 to point 6) and is aligned with the fibre axis (Fig. 10b).

- Distance between two fibres: due to the random positions of points 3 and 4 for each fibre, the distance between two fibre axes $D_{f}$ should be greater than the sum of $2 L_{2} \sin (\alpha)$ and diameter of fibre's crosssection. For better understanding, Fig. 10b shows the transparent grey cylinder representing the critical surface, on which these points and the corresponding line segment are possibly produced.

- Offset: in agreement with the assumption used for $D_{f}$ and similar to the spiral fibre model, the offset value can be sat as $D_{o}+L_{2} \sin (\alpha)$.

\subsubsection{Computer algorithm}

Three modules introduced in the algorithm of the spiral fibre model are also used here. But they are modified in agreement with the geometry of hooked-end fibre. The modifications include the configurations of $D_{m}, D_{o}$, $D_{f}, L_{f}$ and $N_{f}$ as the inputs to Axis Generator. According to the geometric features provided, Single-Fibre Maker generates a model where the positions of the points 1,2, 5 and 6 are constant while the points 3 and 4 are randomly produced for each fibre. So, the outputs comprise $N_{f}$ models in contrary to the spiral fibre where only one fibre is modelled. The fibres produced are exported to Assembler. As an example, Fig. 10c displays the final model produced with 50 fibres translated and rotated by Assembler based on the fibre vectors, and Fig. 11 shows an integrated algorithm to yield the nodes and element matrices for straight, spiral and hooked end fibres. The algorithm suggested gives an insight into the general procedures. Such an algorithm could be consistently modified or redesigned in different cases.

\section{Particle insertion}

Particles (coarse aggregates) are added to the fibrous model using a technique which deals with the Voronoi tessellation method. The technique is based on the generation of Voronoi cells using the FGPs as the seed points. It makes the cell be systematically positioned against the fibres. For the spiral and hooked-end fibres, the FGPs of the fibre axes are used. In the following, the method of particle insertion is only described concerning the straight fibres for avoiding complication. However, it can be accordingly broadened for the spiral and hooked-end fibres. Fig. 12a schematically shows how the particles are formed through four steps:

Step 1. The domain is firstly tessellated and each Voronoi cell encloses one node of the fibres. The geometric relationship between the Voronoi cells, the $1 \mathrm{D}$ elements and the corresponding nodes produced under the rule of connectivity were discussed earlier and highlighted in Fig. 3c.

Step 2. Some Voronoi cells become candidate for simulating particles based on any selection criteria. The rest of them are removed from the model. For example, one or both cells, which enclose the endpoints, can be selected for each fibre (Fig. 12a). 
Step 3. The fibres can be trimmed by either two approaches as indicated in Fig. 12b. Firstly, the endpoint enclosed is moved through the element axis to the out (or on the surface) of cells with the minimum displacement of $L_{e} / 2$. Secondly, the 1D element attached to the node is deleted. In Fig. 12a, the endpoints are moved to the surface of the cells as highlighted in the magnified visualizations.

Step 4. This step can be extended into another module such as splining, shape/size configurations [31] and ITZ generation which will be explained in the next step.

Some key points should be considered through the modelling process: (1) Referring to Fig. 12b, the fibres and the related Voronoi cells do not intersect after trimming in Step 3. Because the distance between the new closest endpoint on the trimmed fibre to the face of the polyhedral cell selected is within the range from zero to $L_{e} / 2$. (2) The cells containing the child points are not selected in Step 2 as it will cause discontinuity through the fibre structure in Step 3. (3) Due to trimming process, the length of the fibre is reduced. Therefore, the length of the original fibre needs to be set up with attention to the deducted length for the fibre generation. For this, the original fibre should be equal to $L_{f}+L_{\text {deducted }}$ (4) In Step 3, if the element is supposed to be deleted, the original total number of elements on a fibre should be at least one more than the number of deleted ones. Otherwise, the fibre is completely removed from the model. After Step 4, the output of is the matrices of nodes and triangle elements of particle labelled by ID numbers.

\section{Modelling the ITZ}

\subsection{Boundary of ITZ}

The method to simulate the ITZ that surrounds each coarse aggregate (particle) uses the particle structures previously generated. It is mainly based on the scaling technique [31] but with modification. The modelling process includes two steps: (1) The triangulated surface of a particle is duplicated by reproducing the matrix of vertices (or nodes). (2) The duplicated particle is expanded with reference to the centroid position $C$, which is obtained by averaging the positions of all vertices. The new positions of vertices $V_{i}^{\text {Scaled }}$ are calculated in the Cartesian coordinate system using the following equation:

$$
V_{i, j}^{\text {Scaled }}=C_{j}+q_{i}\left(V_{i, j}^{\text {Original }}-C_{j}\right), \quad i=1 \text { : number of cell vertices, } j=x, y, z
$$

where the expansion factor $q_{i}$ is specified relative to the thickness of ITZ for each vertex as:

$$
q_{i}=1+\frac{r_{i}}{d_{I T Z}}
$$

where $d_{I T Z}$ denotes the thickness of ITZ which is constant and $r_{i}$ is the original distance between the vertex and the centre.

In contrast with the shrunk factor in the scaling system presented in [31], the expansion factor is not uniformly applied for all vertices in order to keep the thickness consistent among two surfaces. Fig. 13a exemplifies how a particle is schematically scaled up respect to its original model. All vertices are translated along a vector that connects each the centroid to the vertex with the displacement equal to the ITZ thickness. ITZ is basically simulated as an interlayer between the surfaces of the original particle and the scaled one with the same centre.

The method is efficient since the value of ITZ thickness relative to $r_{i}$ is practically large enough and/or the particle can be mainly depicted as a convex shape. Since local concavity can cause a problem in modelling 
thin ITZ. Fig. 13b exemplifies a geometry with the concave feature. It is potential to create the self-intersecting of the ITZ boundary and overlapping with itself. To overcome this issue, the MATLAB function of 'convhulln' is employed to create a convex layer around the particle. The function computes the convex hull of the vertices. The solution presented is computationally reasonable and consistent with this model, although it can be replaced by other approaches to approximate the offset surfaces with higher accuracy. In a FE model, if ITZ thickness is extremely low in comparison with the particle size, ITZ might be better to be simulated using zerothickness interface elements instead of solid elements.

\subsection{Modification on particle generation}

The boundary of particles is expanded to create the ITZ model, which may overlap with other adjacent components like other particles or fibres. To avoid intersecting, the algorithm presented in Fig. 12a needs to be modified by shrinking the original Voronoi cells after Step 3 and before Step 4. Fig. 14 clarifies the modification proposed with attention to the presence of ITZ. The initial Voronoi cells are scaled down using the similar method for expanding the particles to pre-set a margin equal to $d_{I T Z}$ for the model. The margin ensures the extended particles in the stage of ITZ simulation are remained enclosed in the original Voronoi cells with no intersection. In general, the sum of the expansion and shrunk factors through the modelling process is not allowed to be greater than one.

\section{Mortar}

So far, three main phases in FRC including the fibre, particle (coarse aggregate) and ITZ have been replicated. The output produced by each module comprises a set of matrices related to the nodes position, the elemental connectivity and the phase IDs. The data can be exported from MATLAB with a format consistent with the common FE packages for computational modelling of different properties of FRC. The mesh boundaries of the cubic domain, particle and ITZ are exported as STL file which is one of the most accepted formats. It is used for the triangular representation of 3D surface geometry. The file regarding fibre is simply written with text-based DAT file. By the aid of the auto-mesh module available in software, e.g. ABAQUS or ANSYS, solid elements are fabricated among the surfaces. Also, the mesh is in good agreement with the connectivity of the $1 \mathrm{D}$ elements. So, the fibres are properly embedded into the mesh structure. Although, it should be noted that the geometry and mesh generation are not fully dependent. Since the triangle or 1D elements can be modified for a better mesh with the desired size after the mesostructure generation. For example, the coarse elements can be divided into smaller ones, if a finer mesh is required. Thus, the mesh quality can be always improved by choosing an adequate technique compatible with the modelling requirements. Fig. 15a and $\mathbf{b}$ illustrate an example of the solid model of four-phase FRC consisting of the tetrahedral elements and the 1D elements. The mortar as the matrix is created by meshing between the external surfaces of the ITZ models and the sides of the cube. For visual comparison, the X-ray CT images of typical structures for irregular aggregates, mortar [38] and fibres with random orientation [23] in the unreinforced and the reinforced concrete are shown in Fig. 15c and d. A close morphological similarity can be observed in terms of irregularity feature and the random distribution between simulation and experiments. In the models shown, the number of aggregates and fibres were intentionally set to be small for better visualisation of the structural features. Thus, the actual samples cannot be quantitively compared with the models. 


\section{Solid version of fibre model}

In some cases, solid fibre models might be required to, for example, investigate damage through the fibre structure in three dimensions. So, a technique is proposed to consistently develop the fibre made by 1D elements by considering a circular cross-section. To build a solid model, the triangulated surfaces representing the fibre boundaries are first generated. As shown in Fig. 16a, two connected 1D elements with the ID numbers of 1 and 2 are used to imply the method which can be likewise employed for all types of fibre. Each node is the centre of a circle with the axis parallel to the axis of the element number one. Some points are uniformly produced on the circle's perimeter and they divide the circumference into an equal number of segments. The number of points on the circle and the radius of the circle are input parameters to the code. These points are labelled sequentially in order of generations through a fibre structure. The ordered attributes facilitate definition of element connectivity for the model as continuous geometry. The function of 'convhulln' is used to generate a closed cylinder with the triangulated surfaces around each $1 \mathrm{D}$ element through the points on the circles. The triangle elements produced on the end faces of the cylinder need to be deleted because: (1) the elements related to the child points' circle do not belong to the surface of the body; (2) the triangle elements on the parent points' circle have low quality. After performing this procedure for all the elements for fibre, an open-ended surface is created. In contrast to what has been done to open the cylinders, it now needs to close the geometry. In this regard, the parent points and the points on the corresponding circles are only used for the triangulation process at each side. All the triangle elements must have one mutual vertex at the centre and two other vertices are the closest adjacent points on the circumference. This triangulation method is planned to provide a set of elements with higher quality. In general, the quality of the mesh surface depends on the length of $1 \mathrm{D}$ elements set earlier and the number of points on the circle. Fig. 16b depicts the solid models of the spiral and hooked-end fibres. In the next stages of modelling, the triangulated surfaces of fibres are treated as same as the particle and the ITZ models.

\section{An example of model implementation}

To show how the model is configured and works, as a case study, the effect of fibre volume fraction on the elastic modulus of concrete is investigated using the FE method. All phases are assumed to be linearly elastic and isotropic. Based on common idea, the side length of RVE should be at least three to five times larger than the maximum aggregate size [39]. Thus, the RVEs are proposed with the dimensions of $D_{m}=50 \mathrm{~mm}$ and $D_{o}=0.5 \mathrm{~mm}$ to guarantee the length required. To configure a model, all the inputs should be consistent with each other and the RVE size. For instance, it is not possible to generate fibres with the length of $30 \mathrm{~mm}$ randomly distributed while the minimum distance between them is $6 \mathrm{~mm}$ and $30 \%$ of RVE is occupied by the coarse aggregates. Another example is about the total number of aggregates, which can vary in a range for a specific sieve aperture in the structure of an unreinforced concrete [40, 41]. But here, this number should be proportionally adjusted based on the sieve-size data and the number of fibres, which itself depends on the size and volume of the fibres. An unnecessary large number of aggregates would generate undesired short fibres, while an insufficient small number of aggregates would lead to a smaller number of fibres than required.

In the previous work [31], it was discussed how the initial size of Voronoi cells can be controlled by the number of seed points and their distribution mode. It was stated that a large enough number and size of cells 
should be produced as a requirement prior to the size adjustment step. In the current modelling approach, the seed points generation are coupled with the fibre generation as stated within Section 2. FGPs play the role of the seed points in the Voronoi tessellation. The number and distribution of FGPs can be controlled by different parameters such as $N_{f}, D_{o}, D_{f}$ and $L_{f}$. Hence, the tessellation can be set up using a wide range of combination of these modelling parameters. Depending on what parameters are fixed, a model can be configured by the rest of parameters. If, for example, a model is constrained with fixed values of $L_{f}, D_{f}$ and the fibre volume fraction, the initial Voronoi cell size can be regulated by $D_{m}$ and number of child points. In the following, the specifications of four phases including fibres, coarse aggregates, mortar and ITZ, FE configurations and results are presented.

\subsection{Phase specifications}

- Fibre: straight and hooked-end fibres are distributed with the random orientations. The fibre volume fractions are set to $0.5 \%, 1 \%$ and $1.5 \%$. For the straight fibres, the original length of the fibre axis and $L_{e}$ are adjusted to $15 \mathrm{~mm}$ and $5 \mathrm{~mm}$, respectively. So, after trimming fibre from the parent points, $L_{f}$ becomes $10 \mathrm{~mm}$ as the deducted length from each endpoint is $L_{e} / 2$. The diameter of the circular cross-section is 2 $\mathrm{mm}$. The aspect ratio is therefore equal to 5, but it has a minor influence on the elastic modulus of SFRC within this range of fibre volume [42]. For the hooked-end fibres, the similar settings as the straight fibre models are used to generate the fibre axes. The lengths of $L_{1}, L_{2}, L_{3}$ and the angle of $\alpha$ are 1.5, 2.24, 5 $\mathrm{mm}$ and $63.44^{\circ}$, respectively. Each line segment of the hooked-end element is used as $1 \mathrm{D}$ element. Under the rule of connectivity for both types of fibre, $D_{f}$ is set to $6.1 \mathrm{~mm}$. A summary of model configurations including material and geometric properties are listed in Table 1. It should be highlighted that $N_{f}$ is an important input parameter in the modelling process since it indicates the number of Voronoi cells. There should be enough number of large cells for the generation and the size configuration of aggregates in the next steps. Thus, the fibre number is initially set up to 62 for the whole models with different fibre volume fractions. Then, the extra fibres are removed for each volume fraction. 62 fibres produce the total number of 248 Voronoi cells with a proper size distribution. Overproduction of fibre might cause the generation of small cells.

- Coarse aggregate: in this study, the coarse aggregates occupy 30\% of RVE volume. A typical size distribution [43] and the elastic constants [44] used for coarse aggregates are listed in Table 2. As mentioned earlier, the method to spline the Voronoi cells and calibrate them based on the sieve analysis was presented in [31]. In this regard, the Voronoi cells splined with two levels of iteration are firstly sorted in descending order of their volume. Then, they are adjusted within three size segments including small $\left(6.8-57 \mathrm{~mm}^{3}\right)$, medium $\left(57-449 \mathrm{~mm}^{3}\right)$ and large $\left(449-1072.5 \mathrm{~mm}^{3}\right)$. Fig. 17a and b exemplify the obtained particle size distribution and the generated geometry models of aggregates respectively after the size configuration. The total number of aggregates adjusted varies between 117 to 123 .

- Mortar and ITZ: mortar is composed of fine aggregates (sands) smaller than $2.36 \mathrm{~mm}$ and cement paste. The values of the elastic properties of mortar and ITZ have been variously analysed and differently reported. Based on some previous studies, the elastic modulus of mortar is in a range of 12 to $35 \mathrm{MPa}[14$, $45,46]$. Regarding ITZ, it is difficult to characterise its local mechanical properties due to the complexity 
of the microstructure [47]. In spite of this, ITZ can be assumed as a homogenous material across its thickness similar to mortar but with weaker mechanical properties [48]. The thickness of ITZ varies from 10 to $50 \mu \mathrm{m}$ for normal concrete [49]. Here, the elastic properties of mortar and ITZ are selected within the range previously reported and the ITZ is simulated with the thickness of $50 \mu \mathrm{m}$ as given in Table 3 . The assigned values enable the efficiency of the simulations to be generally evaluated in respect to the numerical and experimental results in the literature. However, the results are not directly compared to prior research because a specific SFRC is not considered.

For reference, the simulation time is measured for generating such models with the settings mentioned consisting of the triangulated surfaces and 1D element. The times are below 1 min using a laptop Dell (Latitude 7490) configured with Intel Core i7-8650U $1.90 \mathrm{GHz}$ processor and 16.0 GB RAM.

\subsection{Finite element analysis}

- Setup: the static analysis is performed in ABAQUS. To refine the mesh, each 1D element is split into two elements. Two-node linear beam elements (B21) are assigned for the fibre models. Four-node linear tetrahedron elements (C3D4) are used for meshing of the mortar, aggregates and ITZ phases. The cubic RVEs are aligned with Cartesian coordinate axes $x, y$ and $z$. The unidirectional tensile stress of $1 \mathrm{MPa}$ is uniformly applied on one side and the opposite side is fixed. For both types of fibre, 10 of the REVs are generated for each volume fraction of fibre with different random settings. The tensile test is simulated in three directions per RVE. An energy-based method is proposed to obtain equivalent elastic modulus. A heterogeneous RVE model composed of four phases is equal to an isotropic homogeneous RVE of SFRC if the external energies of two systems are equivalent under similar loading conditions. Therefore, the equivalent elastic modulus $E_{e q}$ can be calculated as follows:

$$
\left\{\begin{array}{c}
\sigma=E_{e q} \varepsilon \\
\text { External Energy }=\frac{1}{2} \sigma \varepsilon
\end{array}\right.
$$

where the external energy can be obtained as an output of the software package. Eq. (11) governs a linear elastic, homogenous and isotropic system. $\sigma$ is calculated by the sum of the nodal reaction forces on the loaded face dividing by the initial surface area of that face $\left(50 \times 50 \mathrm{~mm}^{2}\right)$. The nominal engineering strain $(\varepsilon)$ is computed by dividing the nodal displacement prescribed to the initial side length of the RVEs $(50 \mathrm{~mm})$.

- The convergence test: it is performed on one of the RVEs containing $1.5 \%$ volume fraction of straight fibre. In this regard, the element number changes from about 2 million to 2.5 million. Each sample is tested in three directions and the standard deviations of $E_{e q}$ varies within the range of 0.058-0.083 GPa. The results shown in Table 4 reveal that the model is not very sensitive to mesh density for the model which has more than 2,348,450 elements.

- Model validation: to validate numerical results, a summary of elastic modulus against the previous experimental study [50] is provided in Table 5. The values of $E_{e q}$ are given as the average of 30 samples for each fibre volume fraction in the current research. The simulation results using the purposed method agree reasonably with the experimental data. The models with fibre volume fractions of $0.5 \%$ and $1 \%$ more accurate than the others. The deviation from the experimental data increases for the RVEs without 
fibre. Better approximations could have been achieved, if the models were regulated in accordance with the specific SFRC and more accurate input parameters.

- Results and discussion: as expected and shown in Fig. 18, $E_{\text {eq }}$ increases with the increase of fibre volume fraction. It can be found that the elastic modulus of the models containing hooked-end fibres is about 100 MPa less than the models containing the straight ones. It can be interpreted as the effect of the fibre geometry on the elastic behaviour of SFRC in such random mesostructure. The hooked-end fibres induce different stress regime through the model compared to the straight fibres. One of the differences can be highlighted by the analysis of local stress concentrations on the fibre structures. Fig. 19 shows the typical Von Mises stress distribution patterns in different phases for the RVEs with the fibre volume fraction of $1.5 \%$ subjected to the tensile loading in $y$-direction. For these RVEs, the values of maximum stress concentrations in the fibre structures are $5.10 \pm 0.43$ and $6.96 \pm 0.75$ for the straight and hooked-end fibres, respectively. According to the simulation results obtained in this study, the stress distribution styles of mortar, ITZ and aggregate are almost similar even with different types of fibre. Probably, the significant higher elastic modulus of fibres would magnify their structural effects on the whole model. Therefore, this effect can be only recognized for the fibre while the mortar, ITZ and aggregate phases do not have such characteristic. The main properties of stress distribution in these phases can be analysed as follows. The maximum values of stress concentrations can be found in the mortar, which seems excessively high. In a physical view, it may be correlated to the interaction of the aggregates, the ITZs and the fibres through the narrow tortuous paths created in the mortar. This effect would be magnified at the nodes around the sharp points and edges of the aggregate's surfaces and fibres while the fibres are perfectly bonded to the mortar. In the simulation view, it would be due to the computational errors induced by the very small size and lowquality of some elements. If so, and if the number of the nodes with wrong values is large, the model yields unreliable results, especially in damage analysis based on stress concentration. Although, its impact might be less on the numerical results if a less mesh sensitive approach like the fracture-based energy method is used. As seen in Fig. 19b, the stress distribution pattern in the ITZ phase with the lowest elastic modulus is mainly affected by the load direction. The stress level on the surfaces perpendicular to the loading direction is greater than the parallel ones. No specific pattern can be distinguished for the coarse aggregates. For all RVEs, the maximum stress changes in a range about 15-27, 5.5-7 and 6.5-8.5 MPa for mortar, aggregate and ITZ, respectively.

\section{Conclusions}

In this study, an advanced model is presented for the complex 3D mesostructure of steel fibre reinforced concrete (SFRC) composed of four phases including fibre, coarse aggregates, mortar and interfacial transition zone (ITZ) between aggregates and mortar. Each phase has a significant influence on the properties of SFRC. The relationship of the Delaunay triangulation and Voronoi tessellation as the simulation key is prescribed to resolve the intersection problem, which has been always an obstacle in the developing such stochastic models. Despite the randomness, the systematic distribution allows simulating fibres even with complex geometries like spiral and hooked-end beside the irregular shaped aggregates. The programmability of the framework and 
controllability of different features enable to generate an ideal mesostructure model, by achieving the following objectives:

- Developing a platform to model steel fibres with different profile shapes;

- Controlling size distribution and orientation through fibrous structure;

- Constructing fibres with different types of elements to be compatible with various case studies;

- Generating irregular aggregates with natural geometric characteristics;

- Configuring aggregates size distribution based on experimental data;

- Easy simulation of ITZ with less model complexity;

- Maintaining computational cost low while increasing simulation fidelity in a fully random system.

Moreover, another important feature is revealed based on the morphological analogy between aggregate and voids in concrete. The similar approach used for irregular aggregates can be employed for simulation of irregular voids in the mesostructure. Thus, voids can be either added as the fifth phase or modelled in a twophase mesostructure where fibres and voids have a longer length-scale feature than aggregates and ITZs. It makes this framework even more comprehensive, flexible and effective in modelling fibre reinforced concrete.

To show the performance of the approach proposed, the geometry models were successfully applied to the FE analysis of the elastic modulus of SFRC in the comparative case study between the straight and hookedend fibres with various volume fractions. The advantages of the mesostructure model can be seen through numerical stress analysis. Although, the model capability could have been more highlighted in some cases like damage evaluation. Failure mechanism can be analysed by incorporation of the mesostructure model and a simplified approach such as discrete element method (DEM) too. DEM is an effective tool to study the deformation mechanisms and the contact interaction of granular media [51-53]. In the ongoing project, the fracture behaviour of SFRC under impact and fatigue loading is being studied using the developed models, which will be presented in a future publication. It should be mentioned that the nonlinear behaviour was not considered here to avoid making this article unduly lengthy. Also, a simple linear case allowed focusing on the mesostructure model as the main aim of this study without the need for any additional effort for interpretation of a nonlinear problem.

In practice, the SFRC model can be employed in fibre mix and concrete reinforcement design as an effective computer-aided tool, since it ensures the actual effects of the structural features. Thanks to the capabilities of the approach proposed, the real size distribution and irregular shape of aggregates are taken into account with the presence of the fibres, which provides a good estimation on the packing density of SFRC. In the point of view of structural reinforcement, the model can provide reliable data about fracture like crack propagation process, which might be very difficult to capture by the experiments. In addition to the mechanical behaviour, thermal and transport properties can be accurately predicted.

In summary, the variations of the simulation tools presented open many possibilities in the modelling of composites with cementitious matrix. The model is extendable for generating other types of fibres like braided fibre. The future work will be focused on the textile reinforcements by a combination of the current computational approach and the function-based method. The model will be proposed for textile reinforced concrete. 


\section{Acknowledgments}

The authors gratefully acknowledge the financial support of the Engineering and Physical Sciences Research Council (EPSRC), UK via grant EP/R041504/1 "Development of a Novel Self-Healing Composite for Sustainable and Resilient Concrete Infrastructure".

\section{References}

[1] Wriggers P, Moftah S. Mesoscale models for concrete: homogenisation and damage behaviour. Finite Elem Anal Des 2006;42:623-36.

[2] Peng J, Hu S, Zhang J, Cai C, Li L-y. Influence of cracks on chloride diffusivity in concrete: a five-phase mesoscale model approach. Const Build Mater 2019;197:587-96.

[3] Nilenius F, Larsson F, Lundgren K, Runesson K. Computational homogenization of diffusion in threephase mesoscale concrete. Comput Mech 2014;54:461-72.

[4] Wittmann FH. Fracture mechanics of concrete. New York: Elsevier; 1983.

[5] Al-Rousan T, Masad E, Tutumluer E, Pan T. Evaluation of image analysis techniques for quantifying aggregate shape characteristics. Constr Build Mater 2007;21:978-90.

[6] Simeonov P, Ahmad S. Effect of transition zone on the elastic behavior of cement-based composites. Cem Concr Res 1995;25:165-76.

[7] Nilsen AU, Monteiro PJ. Concrete: a three phase material. Cem Concr Res 1993;23:147-51.

[8] Naaman A, Alkhairi F, Hammoud H. Fiber reinforced concrete. Contract 1993;100:205.

[9] Oliver J, Mora D, Huespe AE, Weyler R. A micromorphic model for steel fiber reinforced concrete. Int J Solids Struct 2012;49:2990-3007.

[10] Xu Z, Hao H, Li H. Mesoscale modelling of fibre reinforced concrete material under compressive impact loading. Constr Build Mater 2012;26:274-88.

[11] Garboczi EJ. Three-dimensional mathematical analysis of particle shape using X-ray tomography and spherical harmonics: application to aggregates used in concrete. Cem Concr Res 2002;32:1621-38.

[12] Latham JP, Munjiza A. The modelling of particle systems with real shapes. Philos Trans R Soc Lond Ser A 2004;362:1953-72.

[13] Zhu W, Tang C. Numerical simulation on shear fracture process of concrete using mesoscopic mechanical model. Constr Build Mater 2002;16:453-63.

[14] Huang Y, Yang Z, Ren W, Liu G, Zhang C. 3D meso-scale fracture modelling and validation of concrete based on in-situ X-ray Computed Tomography images using damage plasticity model. Int J Solids Struct 2015;67:340-52.

[15] Yang Z, Ren W, Sharma R, McDonald S, Mostafavi M, Vertyagina Y, et al. In-situ X-ray computed tomography characterisation of 3D fracture evolution and image-based numerical homogenisation of concrete. Cem Concr Compos 2017;75:74-83.

[16] Xu W, Lv Z, Chen H. Effects of particle size distribution, shape and volume fraction of aggregates on the wall effect of concrete via random sequential packing of polydispersed ellipsoidal particles. Phys A 2013;392:416-26. 
[17] Wittmann F, Roelfstra P, Sadouki H. Simulation and analysis of composite structures. Mater Sci Eng 1985;68:239-48.

[18] Bazant ZP, Tabbara MR, Kazemi MT, Pijaudier-Cabot G. Random particle model for fracture of aggregate or fiber composites. J Eng Mech 1990;116:1686-705.

[19] Zhu Z, Xu W, Chen $\mathrm{H}$. The fraction of overlapping interphase around 2D and 3D polydisperse nonspherical particles: theoretical and numerical models. Comput Methods Appl Mech Eng 2019;345:728-47.

[20] Grassl P, Antonelli A. 3D network modelling of fracture processes in fibrereinforced geomaterials. Int J Solids Struct 2019;156:234-42.

[21] Bentz D, Garboczi E, Stutzman P. Computer modelling of the interfacial transition zone in concrete. Interfaces Cement Compos 1993:107-16.

[22] Xu Z, Hao H, Li H. Mesoscale modelling of dynamic tensile behaviour of fibre reinforced concrete with spiral fibres. Cem Concr Res 2012;42:1475-93.

[23] Qsymah A, Sharma R, Yang Z, Margetts L, Mummery P. Micro X-ray computed tomography imagebased two-scale homogenisation of ultra high performance fibre reinforced concrete. Const Build Mater 2017;130:230-40.

[24] Fang Q, Zhang J. Three-dimensional modelling of steel fiber reinforced concrete material under intense dynamic loading. Constr Build Mater 2013;44:118-32.

[25] Su Y, Li J, Wu C, Wu P, Tao M, Li X. Mesoscale study of steel fibre-reinforced ultra-high performance concrete under static and dynamic loads. Mater Design 2017;116:340-51.

[26] Pros A, Díez P, Molins C. Modeling steel fiber reinforced concrete: numerical immersed boundary approach and a phenomenological mesomodel for concrete-fiber interaction. Int $\mathrm{J}$ Numer Meth Eng 2012;90:65-86.

[27] Gal E, Kryvoruk R. Meso-scale analysis of FRC using a two-step homogenization approach. Comput Struct 2011;89:921-9.

[28] Brandt AM. Fibre reinforced cement-based (FRC) composites after over 40 years of development in building and civil engineering. Compos Struct 2008;86:3-9.

[29] Cunha VM, Barros JA, Sena-Cruz J. An integrated approach for modelling the tensile behaviour of steel fibre reinforced self-compacting concrete. Cem Concr Res 2011;41:64-76.

[30] Liang X, Wu C. Meso-scale modelling of steel fibre reinforced concrete with high strength. Constr Build Mater 2018;165:187-98.

[31] Naderi S, Zhang M. An integrated framework for modelling virtual 3D irregulate particulate mesostructure. Powder Technol 2019;355:808-19.

[32] Guibas L, Stolfi J. Primitives for the manipulation of general subdivisions and the computation of Voronoi. ACM Trans Graphics (TOG) 1985;4:74-123.

[33] Aurenhammer F. Voronoi diagrams - a survey of a fundamental geometric data structure. ACM Comput Surveys (CSUR) 1991;23:345-405.

[34] Fortune S. Voronoi diagrams and Delaunay triangulations. computing in euclidean geometry. World Sci. 1995:225-65. 
[35] Eberly DH. 3D game engine design: a practical approach to real-time computer graphics. CRC Press; 2006.

[36] Weisstein EW. Helix. 2003.

[37] Murray G. Rotation about an arbitrary axis in 3 dimensions. 2013.

[38] Ren W, Yang Z, Sharma R, McDonald SA, Mummery PM. Three-dimensional in situ XCT characterisation and FE modelling of cracking in concrete. Complexity 2018;2018.

[39] Van Mier J, Van Vliet M. Influence of microstructure of concrete on size/scale effects in tensile fracture. Eng Fract Mech 2003;70:2281-306.

[40] Häfner S, Eckardt S, Luther T, Könke C. Mesoscale modeling of concrete: geometry and numerics. Comput Struct 2006;84:450-61.

[41] Wang Z, Kwan A, Chan H. Mesoscopic study of concrete I: generation of random aggregate structure and finite element mesh. Comput Struct 1999;70:533-44.

[42] Teng T-L, Chu Y-A, Chang F-A, Chin H-S. Calculating the elastic moduli of steelfiber reinforced concrete using a dedicated empirical formula. Comput Mater Sci 2004;31:337-46.

[43] Hirsch TJ. Modulus of elasticity iof concrete affected by elastic moduli of cement paste matrix and aggregate. J Proc 1962:427-52.

[44] Wang X, Zhang M, Jivkov AP. Computational technology for analysis of 3D meso-structure effects on damage and failure of concrete. Int J Solids Struct 2016;80:310-33.

[45] Lv T, Chen X, Chen G. The 3D meso-scale model and numerical tests of split Hopkinson pressure bar of concrete specimen. Constr Build Mater 2018;160:744-64.

[46] Benkemoun N, Poullain P, Al Khazraji H, Choinska M, Khelidj A. Meso-scale investigation of failure in the tensile splitting test: Size effect and fracture energy analysis. Eng Fract Mech 2016;168:242-59.

[47] Mondal P, Shah SP, Marks LD. Nanoscale characterization of cementitious materials. ACI Mater J 2008;105:174.

[48] Mondal P, Shah S, Marks L. Nanomechanical properties of interfacial transition zone in concrete. Nanotechnology in Construction 3. Springer 2009:315-20.

[49] Zheng J, Li C, Zhou X. Thickness of interfacial transition zone and cement content profiles around aggregates. Mag Concr Res 2005;57:397-406.

[50] Williamson GR. The effect of steel fibers on the compressive strength of concrete. Special Publ 1974;44:195-208.

[51] Cundall PA, Strack OD. A discrete numerical model for granular assemblies. Geotechnique 1979;29:4765.

[52] Nitka M, Tejchman J. A three-dimensional meso-scale approach to concrete fracture based on combined DEM with X-ray 1CT images. Cem Concr Res 2018;107:11-29.

[53] Suchorzewski J, Tejchman J, Nitka M. Discrete element method simulations of fracture in concrete under uniaxial compression based on its real internal structure. Int J Damage Mech 2018;27:578-607. 


\section{Figures}

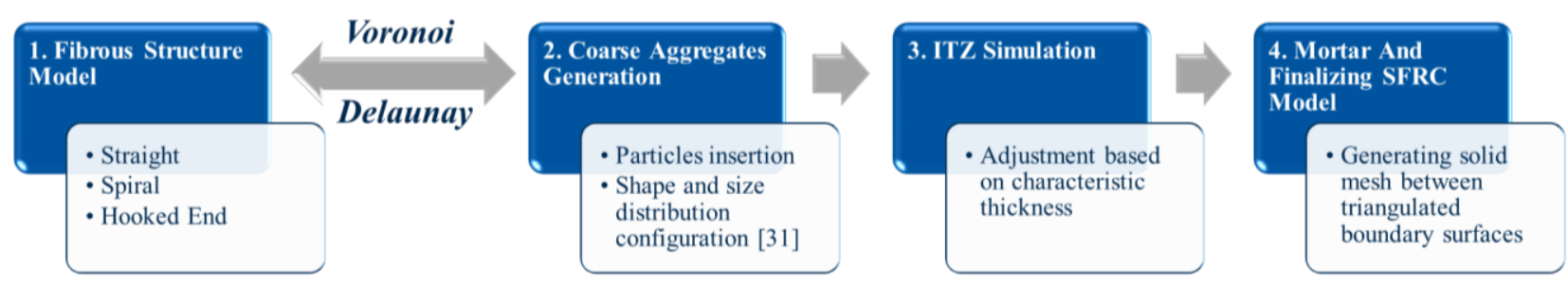

Fig. 1. An overview of the modelling process with the main features in each step.
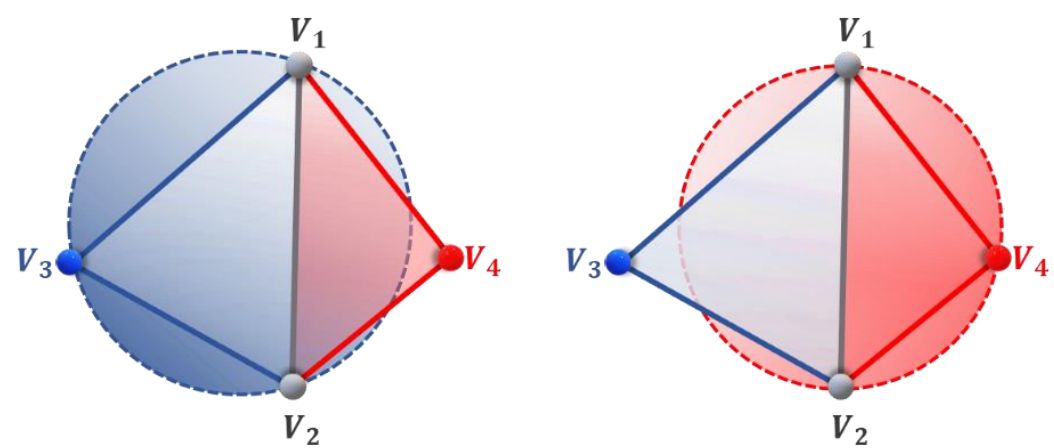

(a)
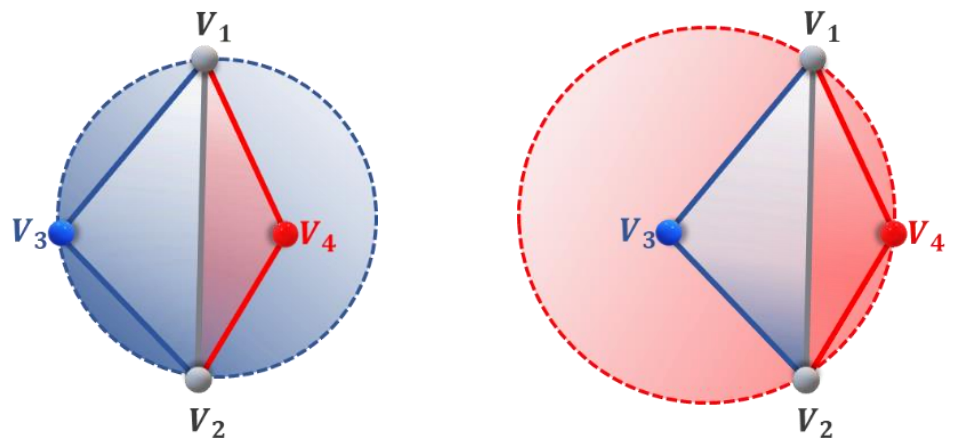

(b)

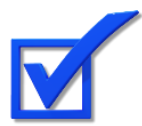

Fig. 2. Delaunay criterion described by two $2 D$ sets of points. (a) The circumcircle associated with the triangle $\left\{V_{1}, V_{2}, V_{3}\right\}$ does not contain $V_{4}$ or any other vertices and similarly, the circumcircle related to the triangle $\left\{V_{1}, V_{2}, V_{4}\right\}$ does not comprise $V_{3}$ or any other vertices. thus, the triangulation is a Delaunay triangulation. (b) The circumcircles are not empty and therefore it does not represent a Delaunay triangulation. 


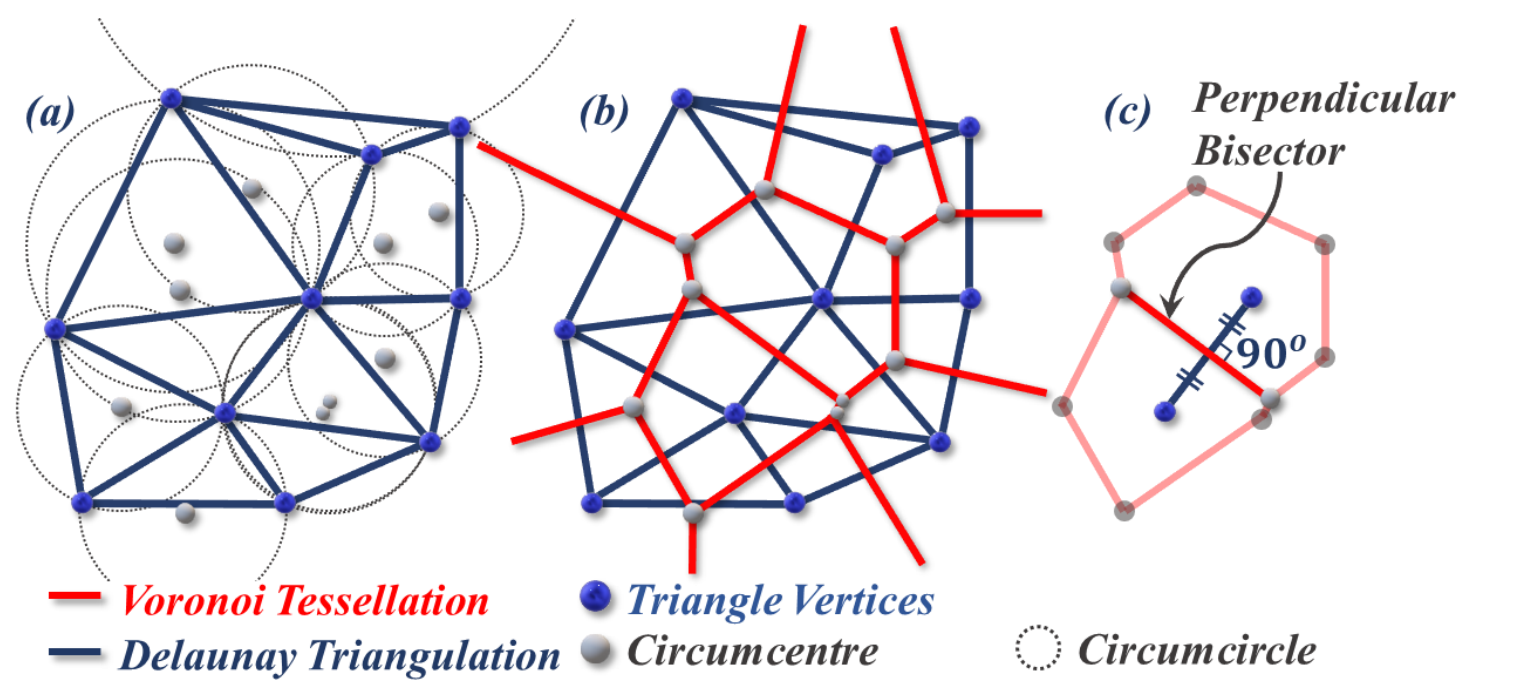

Fig. 3. Relationship between the Delaunay triangulation and the corresponding Voronoi tessellation. (a) The Delaunay triangles associated with the circumcircles and their centres are used for the Voronoi tessellation. (b) The circumcentres are the vertices of the Voronoi cells. (c) The vertices of the Delaunay triangles are the centre of Voronoi cells. The shared edge of two adjacent Voronoi cells is the perpendicular bisector of the line which connects their centres.

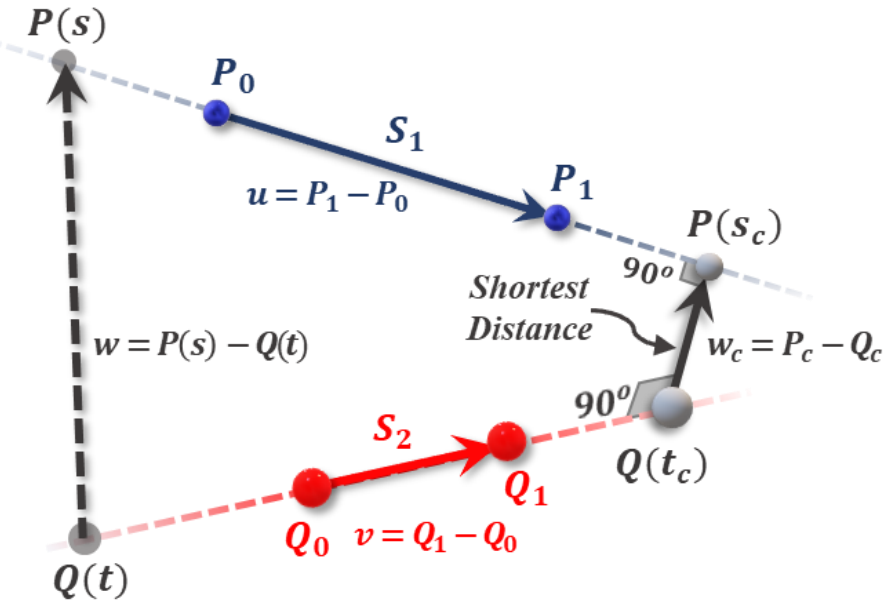

(a)

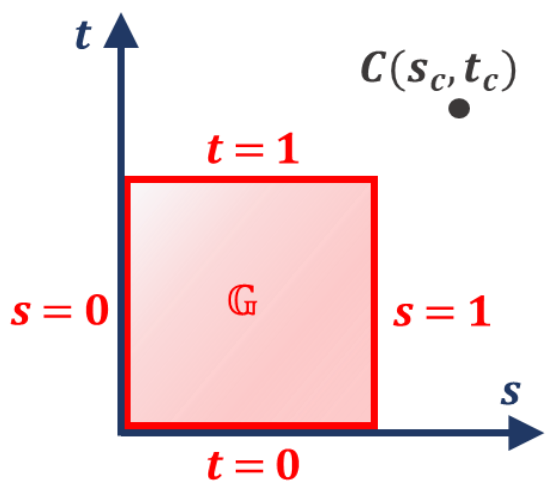

(b)

Fig. 4. (a) Schematic of the determination of the shortest distance between two-line segments based on finding the closest points $Q_{c}$ and $P_{c} .(b)$ The region $\mathbb{G}$ is defined regarding the quadratic function of $|\boldsymbol{w}|^{2}$ and the range of $s$ and $t$. 


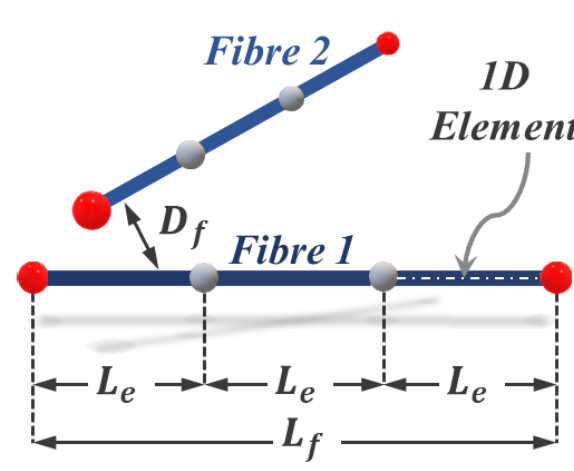

Parent Point

Child Point

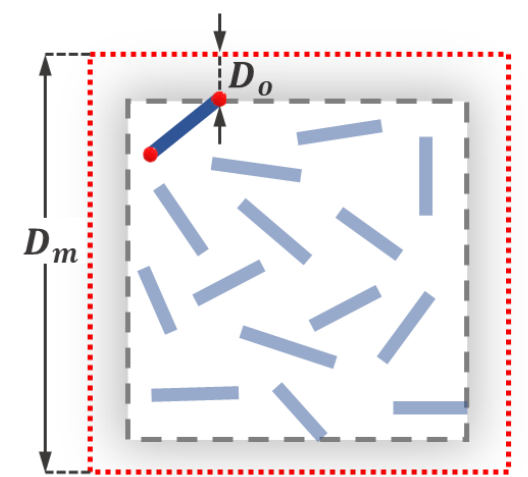

Free Surface Boundary

Fibres

(a)

(b)

Fig. 5. Geometric parameters used in the generation of the straight fibres. (a) A fibre with length $L_{f}$ is defined between the parent points and it can be divided by the child points into equal sublines as a $1 D$ element with length $L_{e}$. The minimum distance between fibres is $D_{f} .(b) D_{m}$ is the domain side length. $D_{o}$ specifies the minimum distance between fibres and the free surface boundaries. In other words, no fibre is generated within the grey zone as schematically displayed.

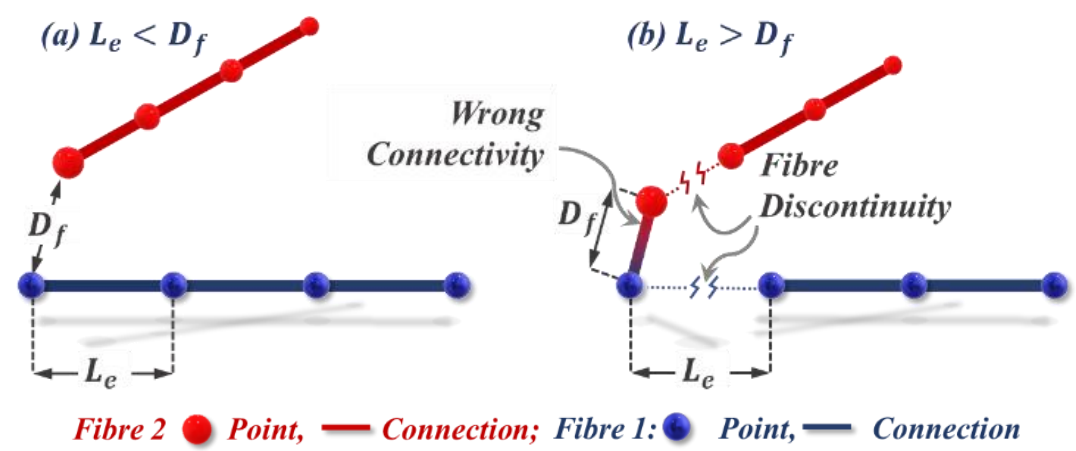

Fig. 6. Two states possibly observed through connecting the points based on the Delaunay criterion. (a) The rule of connectivity implies that the condition of $L_{e}<D_{f}$ must be always satisfied to perfectly connect the corresponding points on each fibre. (b) Otherwise, the wrong link and discontinuity are constructed. 


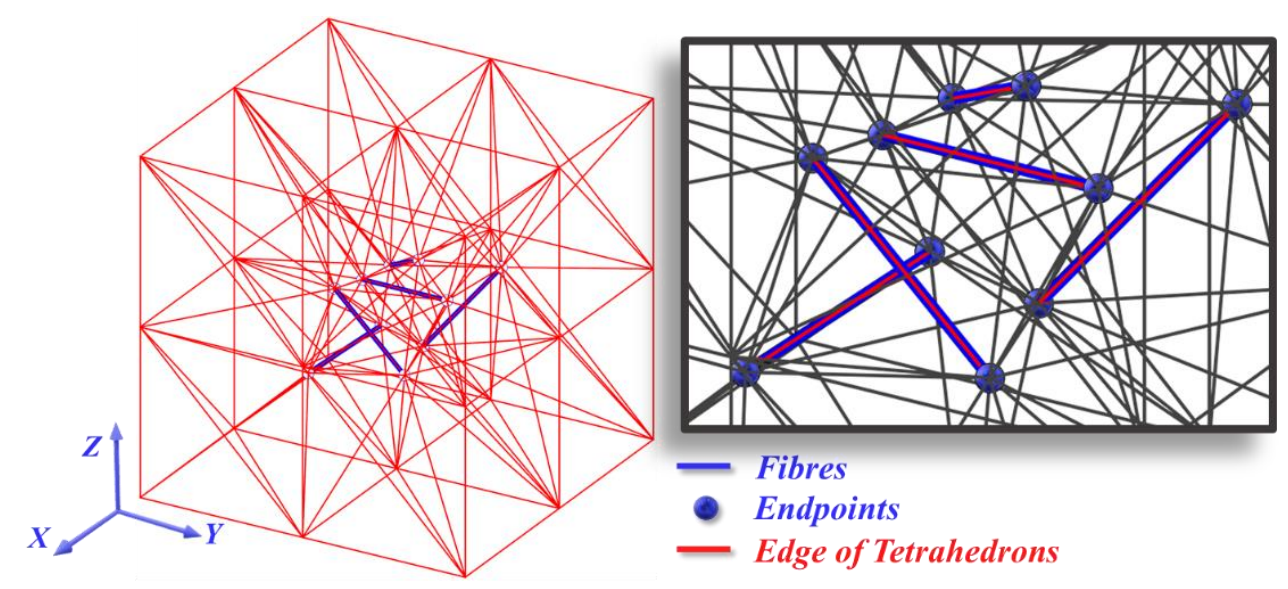

Fig. 7. Fibres aligned with the edges of the tetrahedron elements through the structure of the Delaunay-based mesh. For better visualisation, only a few fibres produced, and they are shown with the magnification of the region of interest.

(a)

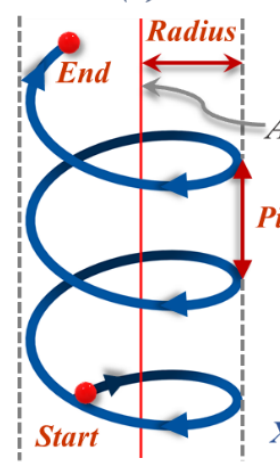

- Parent Point

- Child Point -1D Element OEnd Plane Pitch

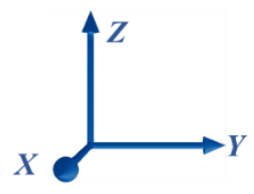

(b)

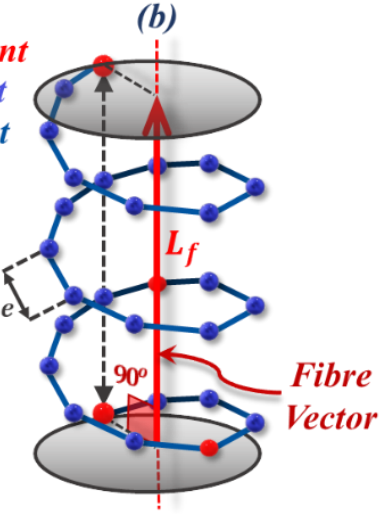

Fig. 8. (a) Circular spiral (helix) curve can be parameterized by radius, pitch and Eq. (6). (b) The spiral fibre model is made by $1 D$ elements with length $L_{e}$ through the parent and child points. The parent points are on a line parallel to the axis. The length of the line represents the fibre length of $L_{f}$. The fibre vector is defined between two end planes as displayed. 


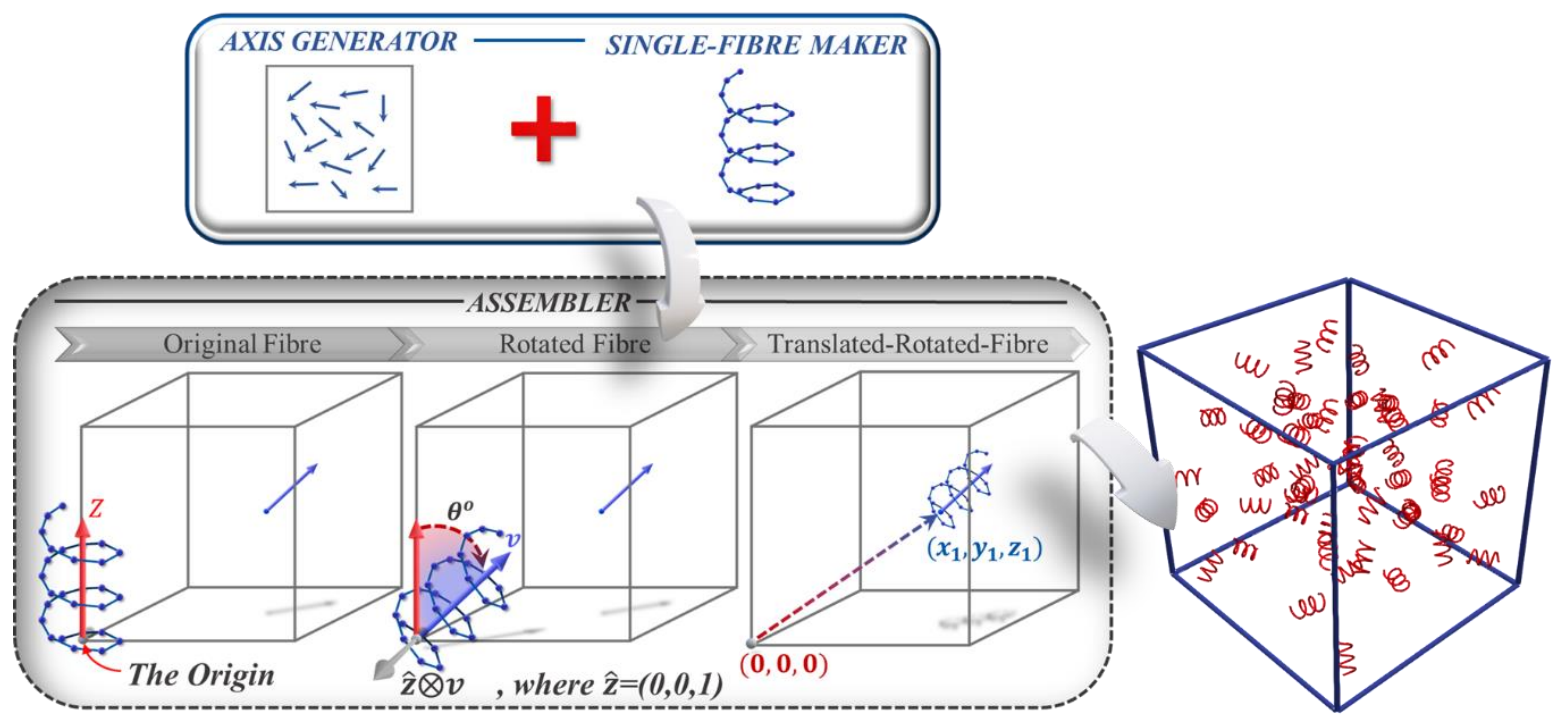

Fig. 9. Algorithm for generating the spiral fibre model based on three interlinked modules including (1) Axis Generator, (2) Single-Fibre Maker, and (3) Assembler. Assembler uses the fibre vectors besides the matrices of nodes and elements. It duplicates the original model, rotates along the axis vector and translates to the position of the corresponding axes. The final model comprised of 50 spiral fibres is illustrated as an example.
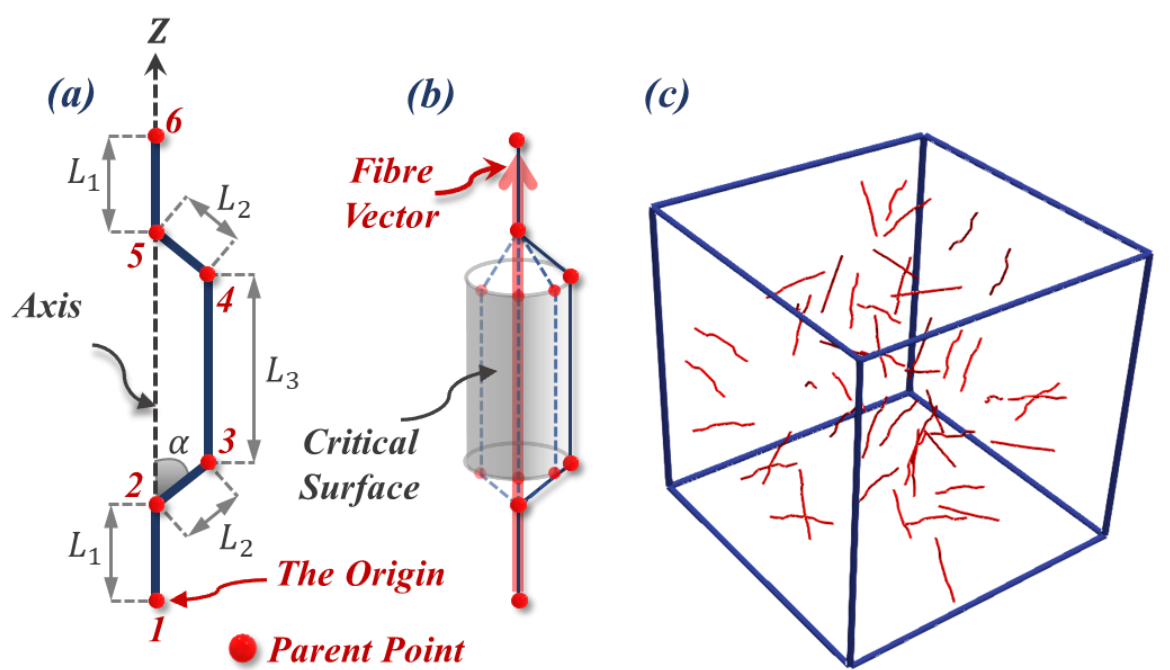

Fig. 10. (a) The geometry of hooked-end fibre model is defined by five-line segments among parent points with three characteristics lengths $L_{1}, L_{2}, L_{3}$ and the angle $\alpha$. The axis of fibre is aligned with $Z-$ axis and point 1 is located at the origin of the coordinate system. (b) The fibre vector connects the endpoints of 1 and 6 . The grey surface of cylinder shows the possible random positions of the line segment between the points 3 and 4 with the radius $L_{2} \sin (\alpha)$ about $Z-$ axis.

(c) A sample of the final model includes 50 hooked-end fibres enclosed in a cubic box. 


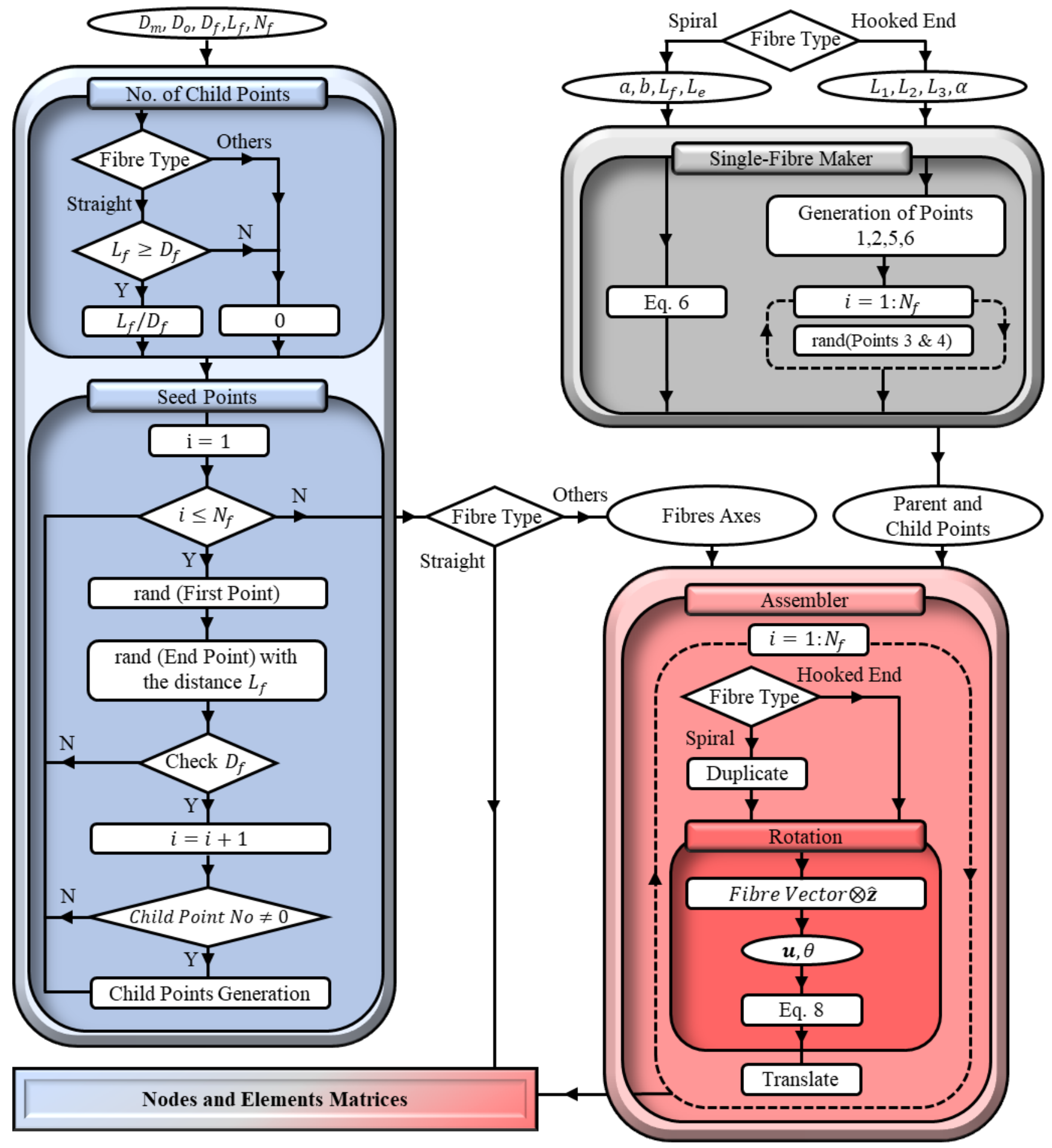

Fig. 11. The integrated algorithm is provided as an example to generate straight, spiral and hooked-end fibres. 

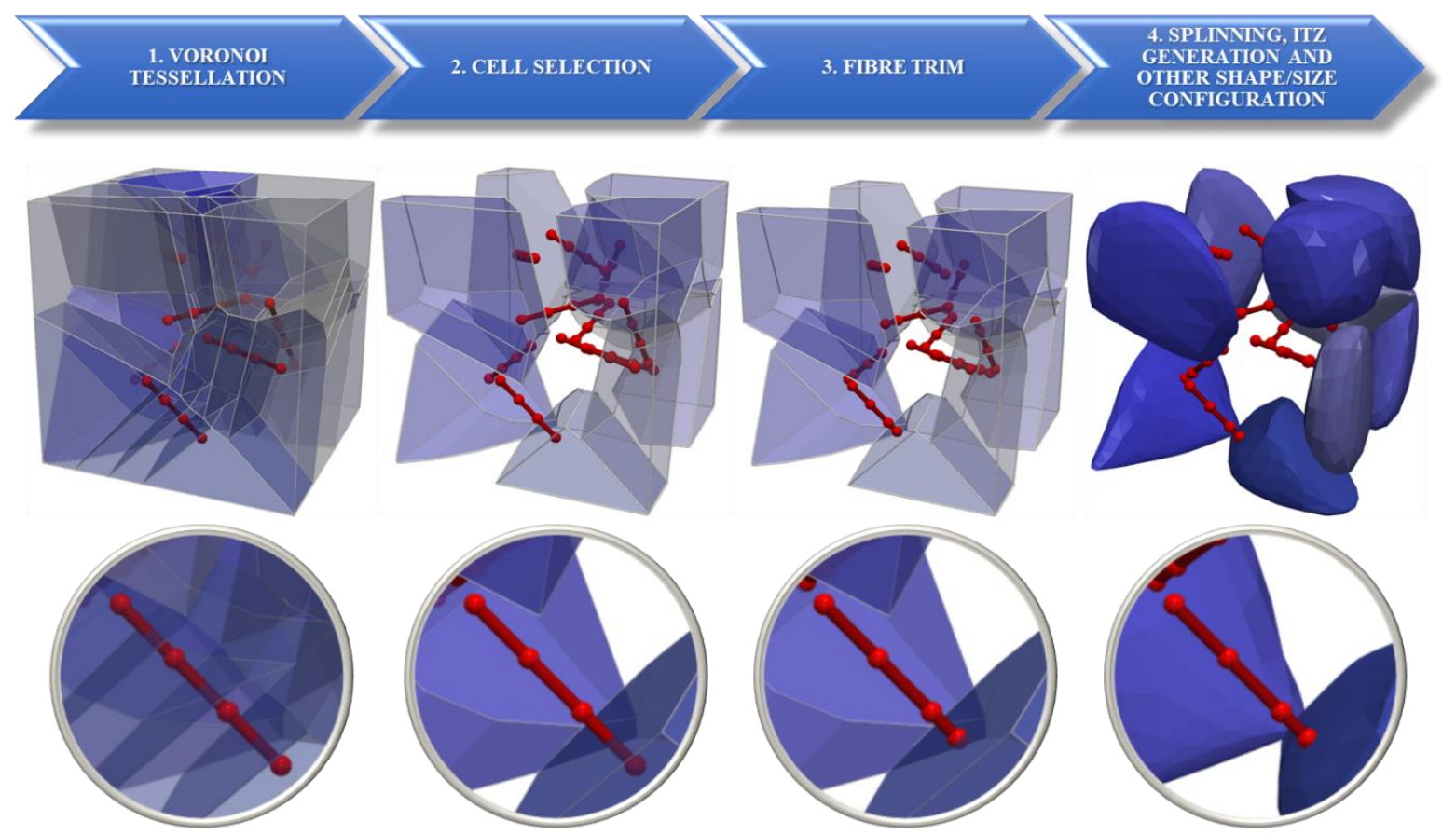

(a)
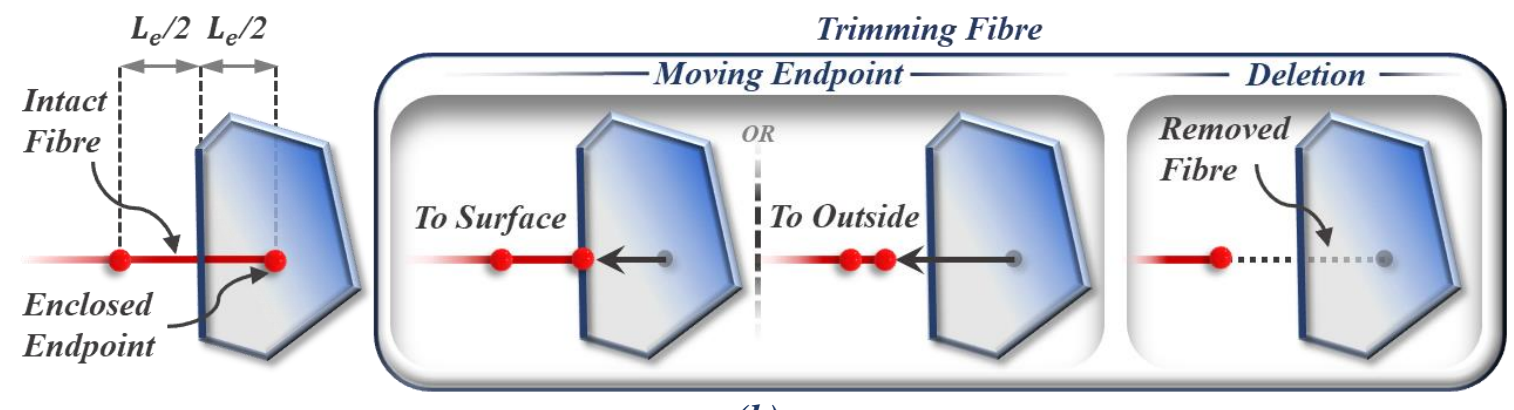

(b)

Fig. 12. (a) The technique to add particles in the fibrous model is mainly based on the generation Voronoi cells by the FGPs as the seed points. For better visualization, one fibre is highlighted in the magnified circle. (b) In agreement with Fig. $3 \boldsymbol{c}$, a fibre can be trimmed by either moving endpoint or deleting fibre. The endpoint can be displaced to the surface or out of the cell.

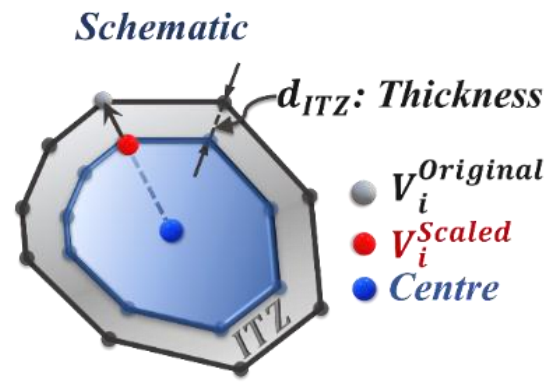

(a)

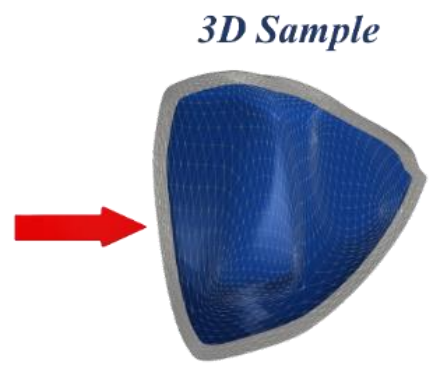

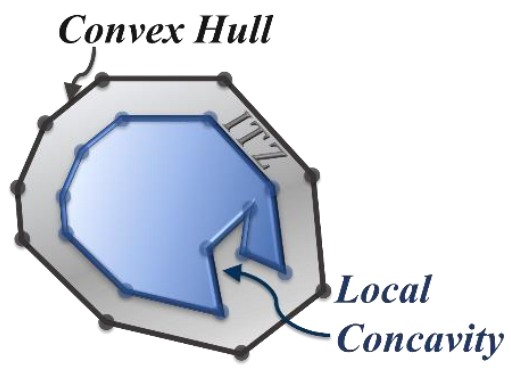

(b)

Fig. 13. (a) ITZ is modelled as an interlayer between the surfaces of the original and the scaled particles. (b) The local concavity can be problematic in the modelling process of the ITZ. In this case, the ITZ boundary can be approximated by the convex hull (grey). 

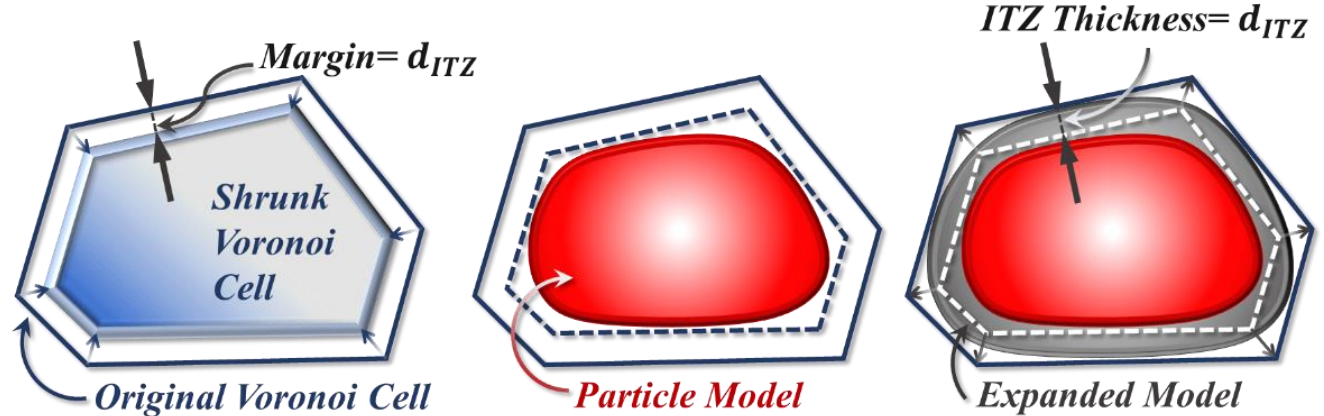

Fig. 14. To modify the algorithm of particle generation, the original Voronoi cells are scaled down to create a margin equal to $d_{I T Z}$. The margin guarantees that the expanded surface of particles will be remained enclosed in the domain of the original Voronoi cell. 


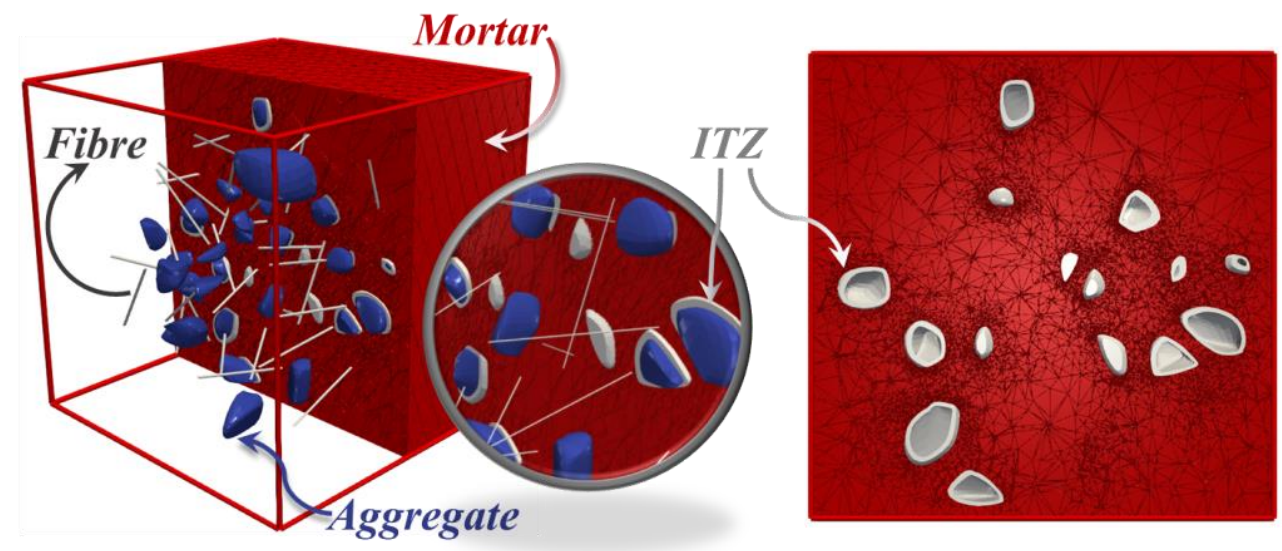

(c)

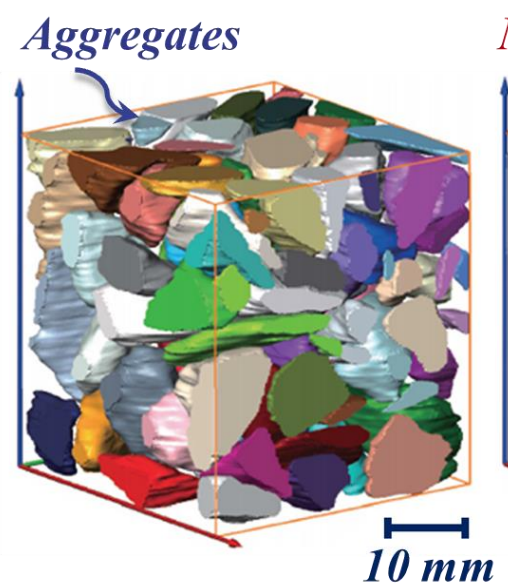

Mortar 2

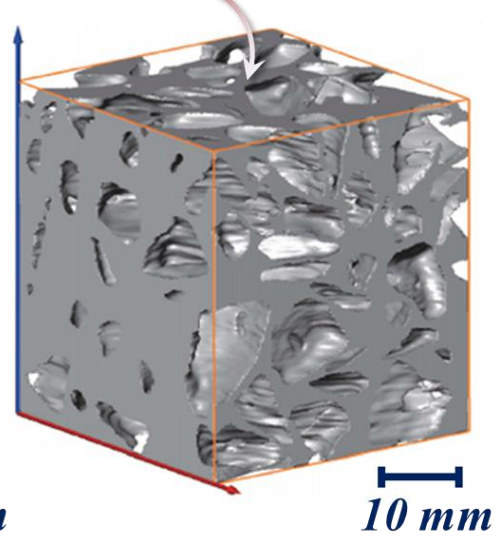

(d)

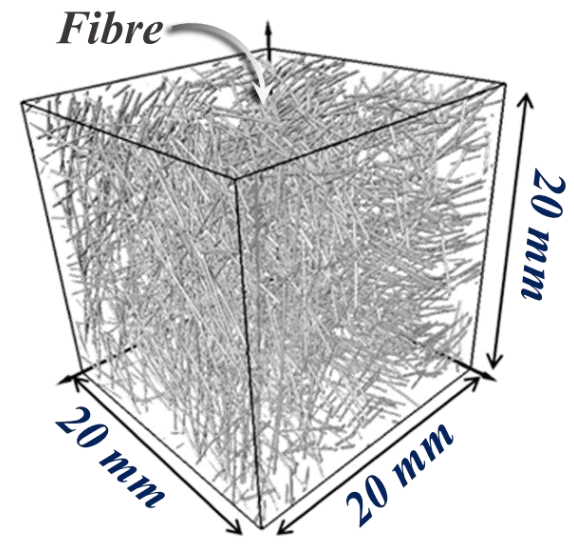

Fig. 15. The mesostructure model explicitly comprises mortar, aggregate, ITZ and fibre. (a) The crosssectional view associated with the magnified image shows the positions of different components relative to each other. (b) To better visualise, the cross-section of the mortar and the ITZ phase are illustrated in the absence of the fibres and the aggregates. The X-ray computed tomography images from the literature are presented to visually compare the model and an actual structure. (c) The typical irregular aggregates in the mortar matrix are shown in the unreinforced concrete [39]. (d) Another image depicts the random distribution of straight steel fibres in the reinforced concrete [40]. The actual samples presented can be only qualitatively compared with the models in terms of shape and distribution attribute, but not regarding size or the number of aggregates and fibres. 


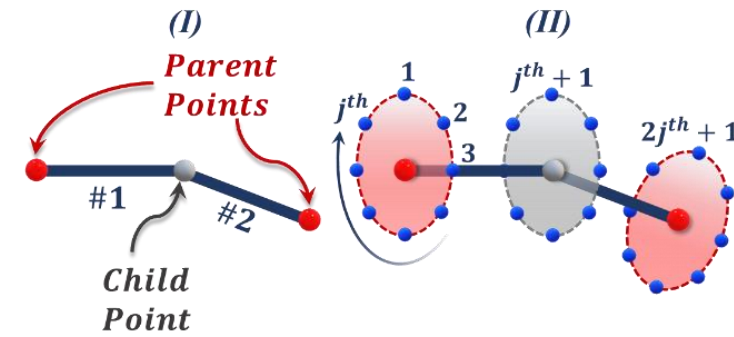

Point
(III)

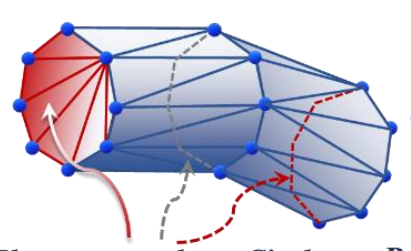

Elements on these Circles needs to be deleted
(IV)

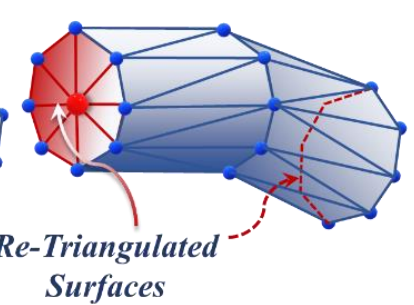

(a)

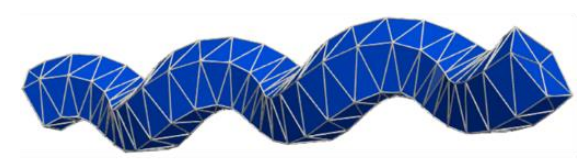

Spiral

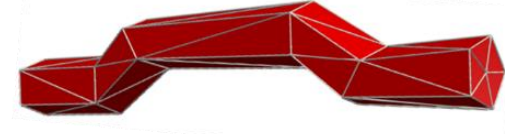

Hooked-End

(b)

Fig. 16. (a) A 1D-element-based fibre model is transformed to a model with a triangulated surface through the procedure which is schematically shown. (b) Two models illustrate the triangular representation of the $3 D$ surface geometries of the spiral and the hooked-end fibre.

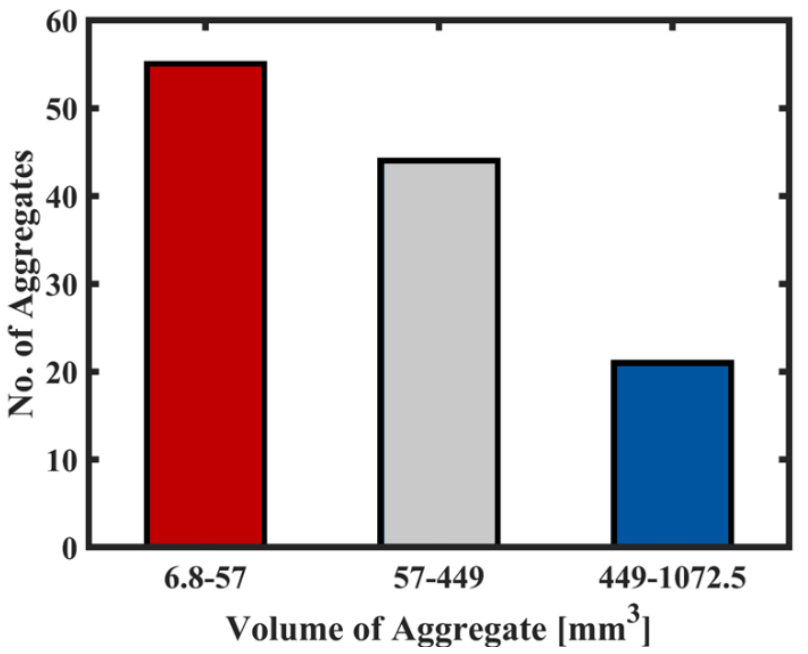

(a)

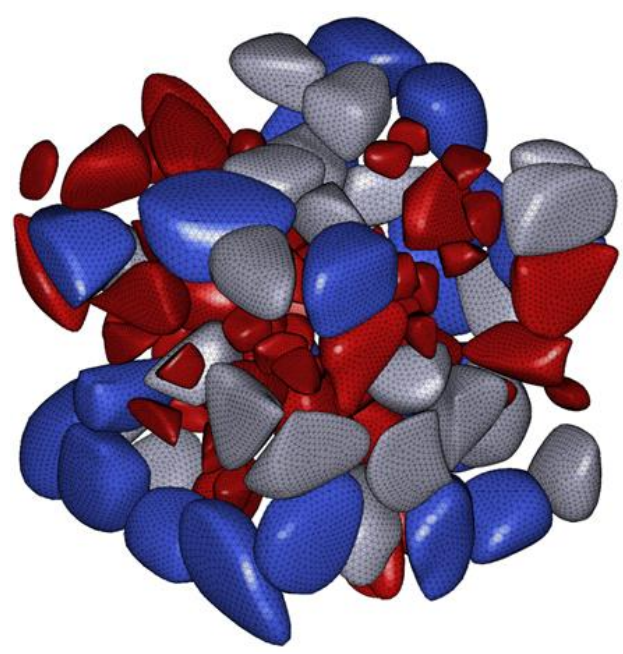

(b)

Fig. 17. (a) The typical aggregate size distribution as the number of aggregates in three volume ranges is illustrated. (b) The geometry model includes the aggregates which are coloured in red, grey and blue according to the size segments shown. 


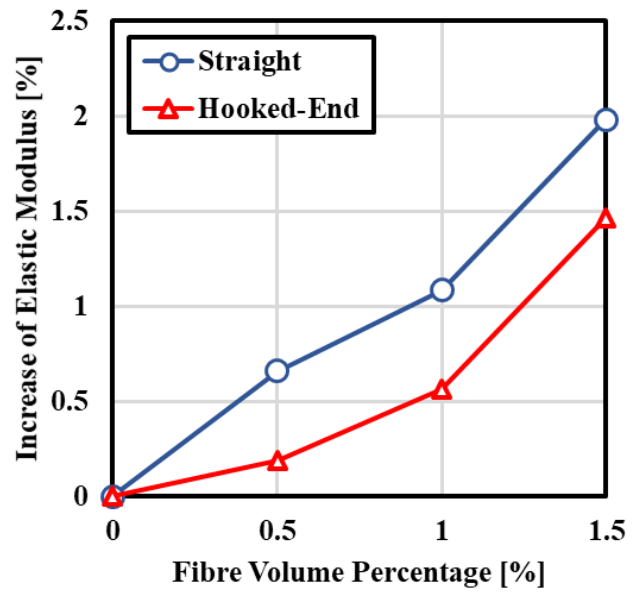

Fig. 18. Evolution of $E_{\text {eq }}$ with respect to the elastic modulus of the concrete model without fibre.

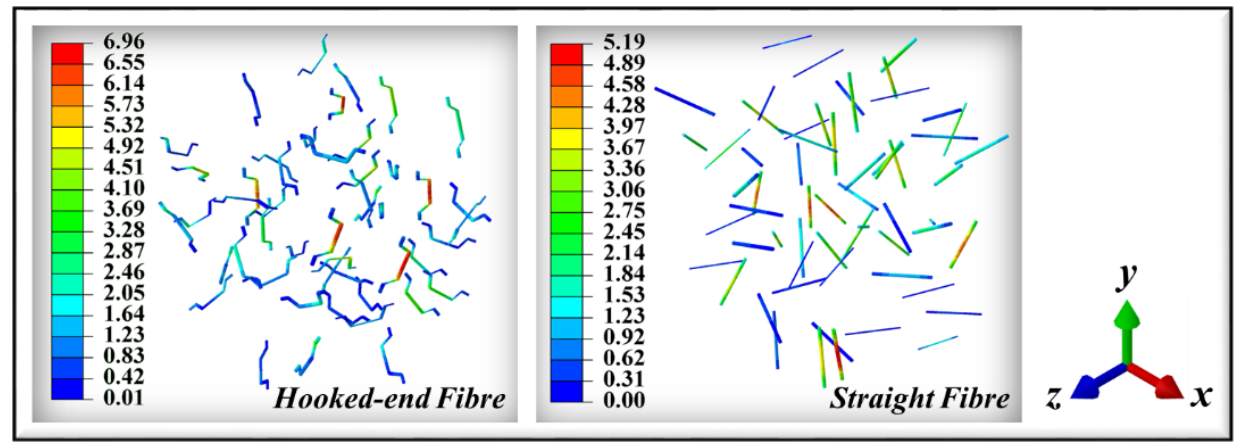

(a)

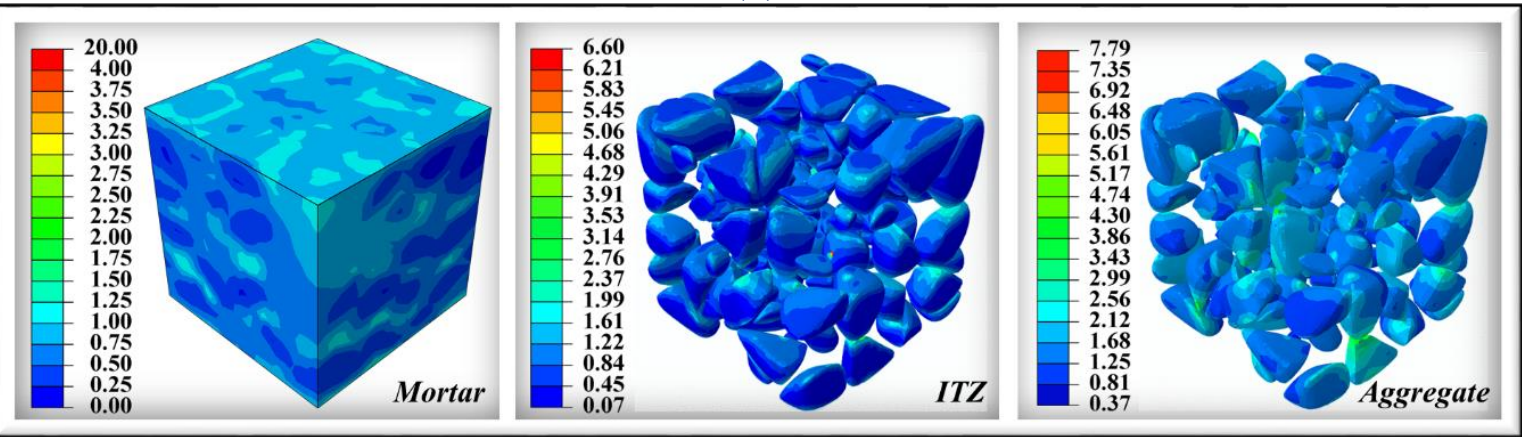

(b)

Fig. 19. Von Mises stress distribution patterns for the models with the volume fraction of $1.5 \%$ for

(a) two types of fibre, and (b) other phases. The stress load is applied in y-direction. All the contours show stress in MPa. The stress contour plot is non-uniformly customized for the phase of mortar to better visualize the stress distribution on the surfaces. 


\section{Tables}

Table 1. Model configurations of fibres.

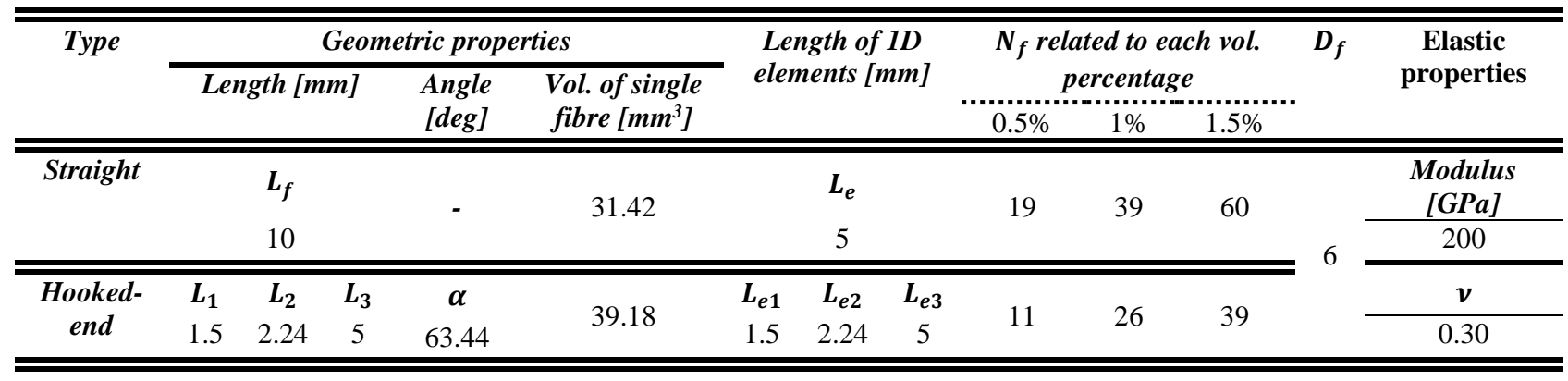

Table 2. Particle size distribution [40] and material properties of coarse aggregate [44] in concrete.

\begin{tabular}{cccc}
\hline \hline \multicolumn{3}{c}{ Particle size distribution } & \\
\cline { 1 - 3 } Sieve size [mm] & $\begin{array}{c}\text { Total percentage } \\
\text { retained [\%] }\end{array}$ & $\begin{array}{c}\text { Total percentage } \\
\text { passing [\%] }\end{array}$ & \\
\hline \hline 12.70 & 0 & 100 & Eodastic properties \\
9.50 & 39 & 61 & 70 \\
4.75 & 90 & 10 & $\boldsymbol{v}$ \\
2.36 & 98.60 & 1.40 & 0.20 \\
\hline \hline
\end{tabular}

Table 3. Material properties of ITZ and mortar.

\begin{tabular}{cccc}
\hline \hline Phase & $\begin{array}{c}\text { Young's modulus } \\
{[\mathrm{GPa}]}\end{array}$ & $\begin{array}{c}\text { Poisson's } \\
\text { ratio }\end{array}$ & $\begin{array}{c}\text { Thickness } \\
{[\mathrm{mm}]}\end{array}$ \\
\hline \hline ITZ & 7.2 & 0.35 & 0.05 \\
\hline \hline Mortar & 14 & 0.20 & - \\
\hline \hline
\end{tabular}

Table 4. Equivalent elastic modulus obtained for the RVEs comprising $1.5 \%$ volume fraction of straight fibre with the different number of elements. Each sample is tested under unidirectional tensile stress applied in three Cartesian directions.

\begin{tabular}{cccccc}
\hline \hline $\begin{array}{l}\text { Stress } \\
\text { direction }\end{array}$ & \multicolumn{4}{c}{$\boldsymbol{E}_{\text {eq }}[\mathbf{G P a}]$ related to each element no. } & $\begin{array}{c}\text { Standard deviation } \\
{[\boldsymbol{G P a}]}\end{array}$ \\
\hline \hline $\boldsymbol{x}$ & 1997902 & 2109880 & 2348450 & 2455556 & \\
\hline $\boldsymbol{y}$ & 21.72 & 21.67 & 21.55 & 21.56 & 0.083 \\
\hline $\boldsymbol{z}$ & 21.66 & 21.63 & 21.51 & 21.52 & 0.076 \\
\hline \hline
\end{tabular}

Table 5. Elastic modulus of SFRC obtained from the current simulation and reported in [50].

\begin{tabular}{|c|c|c|c|c|c|}
\hline \multirow{2}{*}{$\begin{array}{c}\text { Fibre Vol. } \\
\text { Percentage [\%] }\end{array}$} & \multicolumn{2}{|c|}{ Current Work [GPa] } & \multirow{2}{*}{$\begin{array}{c}\text { Williamson } \\
\text { [GPa] }\end{array}$} & \multicolumn{2}{|c|}{ Discrepancy [\%] } \\
\hline & Straight & Hooked-end & & Straight & Hooked-end \\
\hline 0.5 & $21.33 \pm 0.24$ & $21.23 \pm 0.20$ & 21.20 & 0.61 & 0.16 \\
\hline 1.0 & $21.42 \pm 0.20$ & $21.31 \pm 0.15$ & 21.47 & 0.24 & 0.74 \\
\hline 1.5 & $21.61 \pm 0.13$ & $21.50 \pm 0.11$ & 21.75 & 0.62 & 1.17 \\
\hline 0 & \multicolumn{2}{|c|}{$21.19 \pm 0.15$} & 20.80 & \multicolumn{2}{|c|}{1.89} \\
\hline
\end{tabular}

